\title{
Kinetic theory of long-range interacting systems with angle-action variables and collective effects
}

\author{
Pierre-Henri Chavanis ${ }^{1}$ \\ ${ }^{1}$ Laboratoire de Physique Théorique (IRSAMC), CNRS and UPS, \\ Université de Toulouse, F-31062 Toulouse, France
}

\begin{abstract}
We develop a kinetic theory of systems with long-range interactions taking collective effects and spatial inhomogeneity into account. Starting from the Klimontovich equation and using a quasilinear approximation, we derive a Lenard-Balescu-type kinetic equation written in angle-action variables. We confirm the result obtained by Heyvaerts [Mon. Not. R. Astron. Soc. 407, 355 (2010)] who started from the Liouville equation and used the BBGKY hierarchy truncated at the level of the two-body distribution function. When collective effects are neglected, we recover the Landau-type kinetic equation obtained in our previous papers [P.H. Chavanis, Physica A 377, 469 (2007); J. Stat. Mech., P05019 (2010)]. We also consider the relaxation of a test particle in a bath of field particles. Its stochastic motion is described by a Fokker-Planck equation written in angle-action variables. We determine the diffusion tensor and the friction force by explicitly calculating the first and second order moments of the increment of action of the test particle from its equations of motion, taking collective effects into account. This generalizes the expressions obtained in our previous works. We discuss the scaling with $N$ of the relaxation time for the system as a whole and for a test particle in a bath.
\end{abstract}

\section{INTRODUCTION}

Systems with long-range interactions are numerous in nature. Some examples include self-gravitating systems, two-dimensional vortices, non-neutral plasmas and the Hamiltonian Mean Field (HMF) model 11. The equilibrium statistical mechanics of systems with long-range interactions is now relatively well understood [2]. One important feature of these systems is that they may exhibit ensemble inequivalence and negative specific heats first observed in astrophysics (see [3 [5] for reviews). The out-of-equilibrium properties of these systems are also very interesting [2]. In particular, long-range interacting systems may be stuck in non-Boltzmannian quasi stationary states (QSS) that persist for a very long time before finally reaching the Boltzmann distribution. These QSSs are stable steady states of the Vlasov equation which governs the "collisionless" evolution of the system. In order to understand the different timescales involved in the dynamics, it is important to develop a kinetic theory of systems with long-range interactions. Different kinetic theories have been proposed over the years.

Boltzmann [6] developed a kinetic theory for dilute gases. In that case, the particles do not interact except during hard binary collisions. Boltzmann derived a kinetic equation from which he proved that entropy increases $(H$-theorem) and that the system ultimately relaxes towards the Maxwellian distribution of statistical equilibrium.

Landau [7] derived a kinetic equation for a neutral Coulombian plasma in which the charges interact via a $1 / r$ potential. He started from the Boltzmann equation and considered a weak deflection approximation. Since the dynamical evolution of the system is dominated by weak binary collisions, it is possible to expand the Boltzmann equation in terms of a small deflection parameter $\Delta \ll 1$ and make a linear trajectory approximation. However, this treatment yields a logarithmic divergence at small and large impact parameters. The divergence at small scales is due to the neglect of strong collisions, resulting in large deflections. It can be cured by introducing a cut-off at the Landau length, corresponding to the impact parameter leading to a deflection at $90^{\circ}$. On the other hand, in a neutral plasma, the potential is screened on a distance corresponding to the Debye length [8]. Phenomenologically, the Debye length provides an upper cut-off. Later on, Lenard [9] and Balescu [10] developed a more precise kinetic theory that takes collective effects into account. This amounts to replacing the bare potential of interaction $\hat{u}(\mathbf{k})$ by a "dressed" potential of interaction $\hat{u}(\mathbf{k}) /|\epsilon(\mathbf{k}, \mathbf{k} \cdot \mathbf{v})|$ where $\epsilon(\mathbf{k}, \mathbf{k} \cdot \mathbf{v})$ is the dielectric function. Physically, this means that the particles are "dressed" by their polarization cloud. The original Landau equation, which ignores collective effects, is recovered from the Lenard-Balescu equation by setting $|\epsilon(\mathbf{k}, \mathbf{k} \cdot \mathbf{v})|=1$. However, when collective effects are taken into account, it is found that the logarithmic divergence that appears at large scales in the Landau equation is removed and that the Debye length is indeed the natural upper lengthscale to consider. At about the same time, Hubbard [1] ] developed a test particle approach and derived a Fokker-Planck equation describing the relaxation of a test particle in a bath of field particles. He calculated the diffusion and friction coefficients by evaluating the first and second moments of the velocity deflection and took collective effects into account. The Fokker-Planck equation of Hubbard is recovered from the Lenard-Balescu equation in the "bath" approximation. These kinetic theories lead to a relaxation time towards the Maxwell-Boltzmann distribution of the form $t_{R} \sim(\Lambda / \ln \Lambda) t_{D}$, where $t_{D}$ is the dynamical time (the inverse of the plasma pulsation) and $\Lambda$ the plasma parameter (the number of charges in the Debye sphere). 
Chandrasekhar 12 14 developed a kinetic theory of stellar systems in order to determine the timescale of collisional relaxation and the rate of escape of stars from globular clusters. To simplify the kinetic theory, he considered an infinite homogeneous system. He started from the general Fokker-Planck equation and determined the diffusion coefficient and the friction force (first and second moments of the velocity increment) by considering the mean effect of a succession of two-body encounters. Since his approach can take large deflections into account, there is no divergence at small impact parameters and the gravitational analogue of the Landau length naturally appears in the treatment of Chandrasekhar. However, his approach leads to a logarithmic divergence at large scales that is more difficult to remove in stellar dynamics than in plasma physics because of the absence of Debye shielding for the gravitational force. In a series of papers, Chandrasekhar and von Neumann [15] developed a completely stochastic formalism of gravitational fluctuations and showed that the fluctuations of the gravitational force are given by the Holtzmark distribution (a particular Lévy law) in which the nearest neighbor plays a prevalent role. From these results, they argued that the logarithmic divergence has to be cut-off at the interparticle distance. However, since the interparticle distance is smaller than the Debye length, the same arguments should also apply in plasma physics, which is not the case. Therefore, the conclusions of Chandrasekhar and von Neumann are usually taken with circumspection. In particular, Cohen et al. [16] argue that the logarithmic divergence should be cut-off at the Jeans length 17], giving an estimate of the system's size, since the Jeans length is the gravitational analogue of the Debye length. Indeed, while in neutral plasmas the effective interaction distance is limited to the Debye length, in a self-gravitating system, the distance between interacting particles is only limited by the systems's size. These kinetic theories lead to a relaxation time of the form $t_{R} \sim(N / \ln N) t_{D}$ where $t_{D}$ is the dynamical time and $N$ the number of stars in the system. Chandrasekhar [18] also developed a Brownian theory of stellar dynamics and showed that, from a qualitative point of view, the results of kinetic theory can be understood very simply in that framework. In particular, he showed that a dynamical friction is necessary to reproduce the Maxwell-Boltzmann distribution of statistical equilibrium and that the coefficients of friction and diffusion are related to each other by an Einstein relation (fluctuation-dissipation theorem). This relation is confirmed by his more precise kinetic theory based on two-body encounters. It is important to emphasize, however, that Chandrasekhar did not derive the kinetic equation for the evolution of the system as a whole. Indeed, he considered the Brownian motion of a test star in a fixed distribution of field stars (bath) and derived the corresponding Fokker-Planck equation [96]. This equation has been used by Chandrasekhar [14], Spitzer and Härm [20], Michie 21], King [22], and Lemou and Chavanis 23] to study the evaporation of stars from globular clusters. King [24] noted that, if we were to describe the dynamical evolution of the cluster as a whole, the distribution of the field particles must evolve in time in a self-consistent manner so that the kinetic equation must be an integrodifferential equation. The kinetic equation obtained by King is equivalent to the Landau equation. There is, however, an important difference between stellar dynamics and plasma physics. Neutral plasmas are spatially homogeneous due to Debye shielding. By contrast, stellar systems are spatially inhomogeneous. The above-mentioned kinetic theories developed for an infinite homogeneous system can be applied to an inhomogeneous system only if we make a local approximation. In that case, the collision term is calculated as if the system were spatially homogeneous or as if the collisions could be treated as local. Then, the effect of spatial inhomogeneity is only retained in the advective (Vlasov) term which describes the evolution of the system due to meanfield effects [25, 26]. This leads to the Vlasov-Landau-Poisson system which is the standard kinetic equation of stellar dynamics. To our knowledge, this equation has been first written and studied by Hénon 27]. Hénon also exploited the timescale separation between the dynamical time $t_{D}$ and the relaxation time $t_{R} \gg t_{D}$ to derive a simplified kinetic equation for $f(\epsilon, t)$, where $\epsilon=v^{2} / 2+\Phi(\mathbf{r}, t)$ is the individual energy of a star by unit of mass, called the orbit-averaged Fokker-Planck equation. In this approach, the distribution function $f(\mathbf{r}, \mathbf{v}, t)$, averaged over a short timescale, is a steady state of the Vlasov equation of the form $f(\epsilon, t)$ which slowly evolves in time, on a long timescale, due to the development of "collisions" (i.e. correlations caused by finite $N$ effects or graininess). Cohn [28] numerically solved the orbit-averaged Fokker-Planck equation to describe the collisional evolution of star clusters 97]. His treatment accounts both for the escape of high energy stars put forward by Spitzer [30], and for the phenomenon of core collapse resulting from the gravothermal catastrophe discovered by Antonov 31] and Lynden-Bell and Wood 32] on the basis of statistical mechanics. The local approximation, which is a crucial step in the kinetic theory, is supported by the stochastic approach of Chandrasekhar and von Neumann [15] showing the preponderance of the nearest neighbor. However, this remains a simplifying assumption which is not easily controllable. In particular, as we have already indicated, the local approximation leads to a logarithmic divergence at large scales that is difficult to remove. This divergence would not have occurred if full account of spatial inhomogeneity had been given since the start. The effect of spatial inhomogeneity was investigated by Severne and Haggerty [33], Parisot and Severne [34], Kandrup [35], and Chavanis [36, 37]. In particular, Kandrup [35] derived a generalized Landau equation from the Liouville equation by using projection operator technics. Recently, Chavanis [36, 37] obtained this equation in a simpler manner from the BBGKY hierarchy or from a quasilinear theory, as an expansion in $1 / N$ in a proper thermodynamic limit. This generalized Landau equation is interesting because it takes into account effects of spatial inhomogeneity and memory which were neglected in previous approaches. Since the finite extension of the system is properly accounted for, there 
is no divergence at large scales 98. Furthermore, this approach clearly shows which approximations are needed in order to recover the traditional Landau equation. Unfortunately, the generalized Landau equation remains extremely complicated for practical applications. In addition, this equation is still approximate as it neglects "collective effects" and considers binary collisions between naked particles. As in any weakly coupled system, the particles engaged in collisions are dressed by the polarization clouds caused by their own influence on other particles. Collisions between dressed particles have quantitatively different outcomes than collisions between naked ones. In the case of plasmas, collective effects are responsible for Debye shielding. For self-gravitating systems, they lead to "anti-shielding" and are more difficult to analyze [99]. Some authors like Thorne [39], Miller [40], Gilbert [41, 42], Lerche [43], and Weinberg [44] attempted to take both spatial inhomogeneity and collective effects into account. However, they obtained very complicated kinetic equations that have not found application until now. They managed, however, to show that collective effects are equivalent to increasing the effective mass of the particles, hence diminishing the relaxation time. Since, on the other hand, the effect of spatial inhomogeneity is to increase the relaxation time [34], the two effects act in opposite direction and may balance each other.

The same difficulties (and additional ones) occur for other systems with long-range interactions [2]. For example, the kinetic theory of the HMF model [100] is very complicated. In the homogeneous phase (at supercritical energies) the Lenard-Balescu collision term vanishes [47, 48], like in the case of homogeneous 1D plasmas [49, 50], so the kinetic theory must take higher order correlations (three-body, four-body...) into account. In the inhomogeneous phase (at subcritical energies), we have to face the problems of spatial delocalization discussed above. The kinetic theory of $2 \mathrm{D}$ point vortices [51 53] presents very similar features. Finally, 1D stellar systems are always spatially inhomogeneous and it is not possible to make a local approximation, contrary to the $3 \mathrm{D}$ case.

An important problem in the kinetic theory of systems with long-range interactions is therefore to derive a kinetic equation that takes both spatial inhomogeneity and collective effects into account. A standard tool to deal with spatially inhomogeneous systems is to use angle-action variables. This has been used by many authors in astrophysics in order to solve dynamical stability problems 54 58 or to compute diffusion and friction coefficients in a FokkerPlanck approach [59 66]. In an earlier paper [67], starting from the Klimontovich equation and using a quasilinear approximation, we attempted to derive a Lenard-Balescu-type equation with angle-action variables, taking collective effects into account. However, in the course of the derivation, we made a factorization assumption that was not justified. We had the intuition that this problem might be solved by expanding the density-potential functions on a "good" basis but we did not succeed in developing an adequate formalism. However, we argued that the final kinetic equation that we obtained (see Eq. (37) of [67]) is correct when collective effects are neglected. In a subsequent paper [68], we derived this kinetic equation in a more satisfactory manner without ad hoc assumption. Since collective effects are neglected, this kinetic equation can be viewed as a generalization of the Landau equation, written in angle-action variables, applying to spatially inhomogeneous systems with long-range interactions [101].

In the meantime, Heyvaerts [69] managed to derive a Lenard-Balescu-type kinetic equation with angle-action variables starting from the Liouville equation and using the BBGKY hierarchy truncated at the level of the twobody distribution function (i.e. neglecting three-body correlations). To calculate the collective response, he used a biorthogonal basis of pairs of density-potential functions. This is a relatively classical tool in astrophysics [70], introduced by Kalnajs [71], that we did not know at the time of our papers [67, 68]. Although the derivation given by Heyvaerts [69] is very satisfactory, we will show in the first part of the present paper (Sect. II) that the LenardBalescu-type kinetic equation can also be derived from the Klimontovich approach initiated in [67]. The use of a biorthogonal basis of pairs of density-potential functions solves the shortcomings of our previous derivation. This new derivation may be technically simpler than the one given by Heyvaerts [69]. Furthermore, this derivation is completely parallel to the one exposed in [2, 72, 73] to derive the ordinary Lenard-Balescu equation and in [51, 53] to derive a Lenard-Balescu-type equation appropriate to two-dimensional point vortices. Our approach provides therefore a unified description of kinetic theories for systems with long-range interactions when collective effects are accounted for. This generalizes the kinetic theory presented in [68] when collective effects are neglected. Our quasilinear theory has some similarities with the theories developed by Weinberg [64], Ma and Bertschinger [44], and Pichon and Aubert 65] in astrophysics. However, in our case, the source of noise is due to discreteness (finite $N$ ) effects internal to the system while in [64, 65, 74] it is caused by external sources (perturbations on a galaxy, cosmological environment on dark matter halos...). In the second part of the paper (Sect. III), we consider the relaxation of a test particle in a bath of field particles. Its stochastic motion is described by a Fokker-Planck equation with angle-action variables. We determine the diffusion tensor and the friction force by explicitly calculating the first and second order moments of the increment of action of the test particle from its equations of motion, taking collective effects into account. This generalizes the expressions obtained in our previous works [67, 68], neglecting collective effects. We show the connection between the Fokker-Planck equation describing the relaxation of a test particle in a bath and the kinetic equation describing the evolution of the system as a whole. We also discuss the scaling with $N$ of the relaxation time for the system as a whole and for a test particle in a bath. 


\section{COLLISIONAL EVOLUTiON OF THE SYSTEM AS A WHOLE}

\section{A. The Klimontovich equation}

We consider an isolated system of material particles in interaction moving in a $d$-dimensional space. Their dynamics is described by the Hamilton equations

$$
m \frac{d \mathbf{r}_{i}}{d t}=\frac{\partial \mathcal{H}}{\partial \mathbf{v}_{i}}, \quad m \frac{d \mathbf{v}_{i}}{d t}=-\frac{\partial \mathcal{H}}{\partial \mathbf{r}_{i}},
$$

with the Hamiltonian

$$
\mathcal{H}=\sum_{i=1}^{N} \frac{1}{2} m v_{i}^{2}+m^{2} \sum_{i<j} u\left(\left|\mathbf{r}_{i}-\mathbf{r}_{j}\right|\right)
$$

where $u\left(\left|\mathbf{r}-\mathbf{r}^{\prime}\right|\right)$ is the potential of interaction and $m$ the individual mass of the particles. For an isolated system, the total energy $E=\mathcal{H}$ is conserved. We assume that the potential of interaction decays at large distances like $r^{-\gamma}$ with $\gamma \leq d$. In that case, the potential is said to be long-ranged [2]. Long-range potentials include the gravitational and the Coulombian potentials, corresponding to $\gamma=d-2$.

We introduce the discrete distribution function $f_{d}(\mathbf{r}, \mathbf{v}, t)=m \sum_{i} \delta\left(\mathbf{r}-\mathbf{r}_{i}(t)\right) \delta\left(\mathbf{v}-\mathbf{v}_{i}(t)\right)$ and the corresponding potential $\Phi_{d}(\mathbf{r}, t)=\int u\left(\left|\mathbf{r}-\mathbf{r}^{\prime}\right|\right) f_{d}\left(\mathbf{r}^{\prime}, \mathbf{v}^{\prime}, t\right) d \mathbf{r}^{\prime} d \mathbf{v}^{\prime}$. Using the equations of motion (11) and (2), we find that $f_{d}(\mathbf{r}, \mathbf{v}, t)$ satisfies the Klimontovich [75] equation

$$
\frac{\partial f_{d}}{\partial t}+\left[H_{d}, f_{d}\right]=0
$$

where $H_{d}=v^{2} / 2+\Phi_{d}(\mathbf{r}, t)$ is the exact energy of an individual particle and we have introduced the Poisson bracket

$$
[H, f]=\frac{\partial H}{\partial \mathbf{v}} \cdot \frac{\partial f}{\partial \mathbf{r}}-\frac{\partial H}{\partial \mathbf{r}} \cdot \frac{\partial f}{\partial \mathbf{v}}
$$

The Klimontovich equation (3) contains exactly the same information as the Hamiltonian equations (1)-(2).

We now introduce a smooth distribution function $f(\mathbf{r}, \mathbf{v}, t)=\left\langle f_{d}(\mathbf{r}, \mathbf{v}, t)\right\rangle$ corresponding to an average of $f_{d}(\mathbf{r}, \mathbf{v}, t)$ over a large number of initial conditions. We then write $f_{d}=f+\delta f$ where $\delta f$ denotes the fluctuations around the smooth distribution. Similarly, we introduce $\Phi(\mathbf{r}, t)=\left\langle\Phi_{d}(\mathbf{r}, t)\right\rangle$ and write $\Phi_{d}=\Phi+\delta \Phi$. Substituting these decompositions in Eq. (3), we obtain

$$
\frac{\partial f}{\partial t}+\frac{\partial \delta f}{\partial t}+[H, f]+[H, \delta f]+[\delta \Phi, f]+[\delta \Phi, \delta f]=0
$$

where we have written $H_{d}=H+\delta H$ with $H=v^{2} / 2+\Phi$ and $\delta H=\delta \Phi$. Taking the ensemble average of Eq. (5), we get

$$
\frac{\partial f}{\partial t}+[H, f]=-\langle[\delta \Phi, \delta f]\rangle
$$

Subtracting this relation from Eq. (5) and neglecting the nonlinear terms, we obtain an equation for the perturbation

$$
\frac{\partial \delta f}{\partial t}+[H, \delta f]+[\delta \Phi, f]=0
$$

This linearization corresponds to the quasilinear approximation [2]. It is valid at the order $1 / N$ in a proper thermodynamic limit $N \rightarrow+\infty$ in which the system size scales like $V \sim 1$, the individual mass like $m \sim 1$, the total energy like $E \sim N$, the inverse temperature like $\beta \sim 1$, the dynamical time like $t_{D} \sim 1$, and the potential of interaction like $u \sim 1 / N$ [3]. This is equivalent to putting $1 / N$ in front of the potential energy in the Hamiltonian (Kac prescription). In this thermodynamic limit, the distribution function and the potential are of order $f / N \sim \Phi \sim 1$ and their fluctuations are of order $\delta f / N \sim \delta \Phi \sim 1 / \sqrt{N}$ [37]. Therefore, the term in the right hand side of Eq. (6) is of order $1 / N$ (as compared to the left hand side). For $N \rightarrow+\infty$, it vanishes and we obtain the Vlasov equation

$$
\frac{\partial f}{\partial t}+[H, f]=0
$$


In order to deal with spatially inhomogeneous systems, it is convenient to use angle-action variables [70, 76]. By construction, the Hamiltonian $H$ in angle and action variables depends only on the actions $\mathbf{J}=\left(J_{1}, \ldots, J_{d}\right)$ that are constants of the motion (the conjugate coordinates $\mathbf{w}=\left(w_{1}, \ldots, w_{d}\right)$ are called angles). Therefore, any distribution of the form $f=f(\mathbf{J})$ is a steady state of the Vlasov equation. According to the Jeans theorem, this is not the general form of Vlasov steady states. However, if the potential is regular, for all practical purposes any time-independent solution of the Vlasov equation may be represented by a distribution of the form $f=f(\mathbf{J})$ (strong Jeans theorem). We assume that the system has reached a quasi stationary state (QSS) described by a distribution of the form $f=f(\mathbf{J})$ as a result of a violent collisionless relaxation [77] involving only meanfield effects. Due to finite $N$ effects (granularities, correlations...), the distribution function $f$ will slowly evolve in time. This is similar to the effect of collisions in the theory of gases. Finite $N$ effects are accounted for in the right hand side of Eq. (6) which is similar to the collision term in the Boltzmann equation. This term is expected to drive the system towards statistical equilibrium. Therefore, it is expected to select the Boltzmann distribution among all possible steady solutions of the Vlasov equation. Since this term is of order $1 / N$ or smaller, the effect of "collisions" (granularities, finite $N$ effects, correlations...) is a very slow process that takes place on a timescale $N t_{D}$ or larger. Therefore, there is a timescale separation between the dynamical time $t_{D} \sim 1$ which is the timescale at which the system reaches a steady state of the Vlasov equation through violent collisionless relaxation and the "collisional" time $t_{R}$ at which the system is expected to relax towards statistical equilibrium due to finite $N$ effects. Because of this timescale separation, the distribution function will evolve through a sequence of stationary solutions of the Vlasov equation depending only on the actions $\mathbf{J}$, slowly changing with time due to "collisions" (finite $N$ effects). Hence, the distribution function averaged on a short (dynamical) timescale can be approximated by

$$
f(\mathbf{r}, \mathbf{v}, t) \simeq f(\mathbf{J}, t) .
$$

In that case, the distribution function is a function $f=f(\mathbf{J}, t)$ of the actions only that slowly evolves in time under the effect of "collisions". This is similar to an adiabatic approximation. The system is approximately in mechanical equilibrium at each stage of the dynamics and the "collisions" slowly drive it to thermodynamical equilibrium. The purpose of this paper is to obtain a closed expression for the collision term in action space when the rapid dynamics has been averaged over the angles.

\section{B. Angle-action variables}

The previous decomposition has separated the discrete distribution function $f_{d}$ into a smooth component $f$ that slowly evolves with time and a fluctuating component $\delta f$ that changes more rapidly. When we focus on the evolution of the fluctuations, we can consider that the smooth distribution is "frozen". The smooth distribution $f$ determines a smooth potential $\Phi$ that, we assume, is integrable. Therefore, to analyze the evolution of the fluctuations, it is possible to use angle-action variables constructed with this smooth potential [70, 76]. A particle with coordinates $(\mathbf{r}, \mathbf{v})$ in phase space is described equivalently by the angle-action variables $(\mathbf{w}, \mathbf{J})$. The smooth Hamiltonian equations for the conjugate variables $(\mathbf{r}, \mathbf{v})$ are

$$
\frac{d \mathbf{r}}{d t}=\frac{\partial H}{\partial \mathbf{v}}, \quad \frac{d \mathbf{v}}{d t}=-\frac{\partial H}{\partial \mathbf{r}} .
$$

If we use the variables $(\mathbf{r}, \mathbf{v})$, we find that the dynamics is relatively complicated because the potential explicitly appears in the second equation. Therefore, this equation $d \mathbf{v} / d t=-\nabla \Phi$ cannot be easily integrated except if $\Phi=0$, i.e. for a spatially homogeneous system. In that case, the velocity $\mathbf{v}$ is constant and the unperturbed equations of motion reduce to $\mathbf{r}=\mathbf{v} t+\mathbf{r}_{0}$, i.e. a rectilinear motion at constant velocity. Now, the angle-action variables are constructed so that the Hamiltonian does not depend on the angles w. Therefore, the smooth Hamiltonian equations for the conjugate variables $(\mathbf{w}, \mathbf{J})$ are

$$
\frac{d \mathbf{w}}{d t}=\frac{\partial H}{\partial \mathbf{J}} \equiv \boldsymbol{\Omega}(\mathbf{J}), \quad \frac{d \mathbf{J}}{d t}=-\frac{\partial H}{\partial \mathbf{w}}=\mathbf{0},
$$

where $\boldsymbol{\Omega}(\mathbf{J})$ is the angular frequency of the orbit with action $\mathbf{J}$. From these equations, we find that $\mathbf{J}$ is constant and that $\mathbf{w}=\boldsymbol{\Omega}(\mathbf{J}) t+\mathbf{w}_{0}$. Therefore, the equations of motion are very simple in these variables. They extend naturally the trajectories at constant velocity for spatially homogeneous systems. This is why this choice of variables is relevant to develop the kinetic theory. Of course, even if the description of the motion becomes simple in these variables, the complexity of the problem has not completely disappeared. It is now embodied in the relation between position and momentum variables and angle and action variables which can be quite complicated. In this paper, we shall not consider this problem and we shall remain in action space. 
Since the Poisson bracket is invariant on a change of canonical variables, we have

$$
[H, f]=\frac{\partial H}{\partial \mathbf{J}} \cdot \frac{\partial f}{\partial \mathbf{w}}-\frac{\partial H}{\partial \mathbf{w}} \cdot \frac{\partial f}{\partial \mathbf{J}} .
$$

Now, in terms of the angle-action variables, using the relations

$$
\begin{aligned}
& {[H, \delta f]=\frac{\partial H}{\partial \mathbf{J}} \cdot \frac{\partial \delta f}{\partial \mathbf{w}}-\frac{\partial H}{\partial \mathbf{w}} \cdot \frac{\partial \delta f}{\partial \mathbf{J}}=\boldsymbol{\Omega}(\mathbf{J}) \cdot \frac{\partial \delta f}{\partial \mathbf{w}},} \\
& {[\delta \Phi, f]=\frac{\partial \delta \Phi}{\partial \mathbf{J}} \cdot \frac{\partial f}{\partial \mathbf{w}}-\frac{\partial \delta \Phi}{\partial \mathbf{w}} \cdot \frac{\partial f}{\partial \mathbf{J}}=-\frac{\partial \delta \Phi}{\partial \mathbf{w}} \cdot \frac{\partial f}{\partial \mathbf{J}}}
\end{aligned}
$$

and averaging over the angles, we find that Eqs. (6)-(17) take the form

$$
\begin{gathered}
\frac{\partial f}{\partial t}=\frac{\partial}{\partial \mathbf{J}} \cdot\left\langle\delta f \frac{\partial \delta \Phi}{\partial \mathbf{w}}\right\rangle, \\
\frac{\partial \delta f}{\partial t}+\boldsymbol{\Omega}(\mathbf{J}) \cdot \frac{\partial \delta f}{\partial \mathbf{w}}-\frac{\partial \delta \Phi}{\partial \mathbf{w}} \cdot \frac{\partial f}{\partial \mathbf{J}}=0 .
\end{gathered}
$$

These equations govern the evolution of the smooth distribution function and of the fluctuations in angle and action space at the order $1 / N$.

\section{Lenard-Balescu and Landau-type kinetic equations}

Equations (15) and (16) form the basis of the quasilinear approximation. We shall assume that the fluctuations evolve rapidly compared to the transport time scale, so that time variation of $f(\mathbf{J}, t)$ and $\boldsymbol{\Omega}(\mathbf{J}, t)$ can be neglected in the calculation of the collision term (Bogoliubov ansatz). In other words, the distribution function can be regarded as constant in Eq. (16) because it evolves on a (relaxation) timescale which is much longer than the (dynamical) time required for the correlation function $\langle\delta f \delta \Phi\rangle$ to reach an equilibrium for a given value of $f$. This adiabatic hypothesis is valid for $N \gg 1$. For brevity, we shall omit the variable $t$ in $f$ and $\Omega$. It will be restored at the end, when we take into account the time dependence of the smooth distribution function through the kinetic equation (15). We also assume that the distribution function $f$ remains Vlasov stable, so it evolves under the sole effect of "collisions" and not because of dynamical instabilities. In that case, Eqs. (15)-(16) can be solved with the aid of Fourier-Laplace transforms and the collision term can be explicitly calculated. We present below the calculations that lead to the Lenard-Balescu-type equation (56) for spatially inhomogeneous systems.

The Fourier-Laplace transform of the fluctuations of the distribution function $\delta f$ is defined by [102]:

$$
\delta \tilde{f}(\mathbf{k}, \mathbf{J}, \omega)=\int \frac{d \mathbf{w}}{(2 \pi)^{d}} \int_{0}^{+\infty} d t e^{-i(\mathbf{k} \cdot \mathbf{w}-\omega t)} \delta f(\mathbf{w}, \mathbf{J}, t) .
$$

This expression for the Laplace transform is valid for $\operatorname{Im}(\omega)$ sufficiently large. For the remaining part of the complex $\omega$ plane, it is defined by an analytic continuation. The inverse transform is

$$
\delta f(\mathbf{w}, \mathbf{J}, t)=\sum_{\mathbf{k}} \int_{\mathcal{C}} \frac{d \omega}{2 \pi} e^{i(\mathbf{k} \cdot \mathbf{w}-\omega t)} \delta \tilde{f}(\mathbf{k}, \mathbf{J}, \omega),
$$

where the Laplace contour $\mathcal{C}$ in the complex $\omega$ plane must pass above all poles of the integrand. Similar expressions hold for the fluctuations of the potential $\delta \Phi(\mathbf{w}, \mathbf{J}, t)$. If we take the Fourier-Laplace transform of Eq. (16), we find that

$$
-\delta \hat{f}(\mathbf{k}, \mathbf{J}, 0)-i \omega \delta \tilde{f}(\mathbf{k}, \mathbf{J}, \omega)+i \mathbf{k} \cdot \mathbf{\Omega} \delta \tilde{f}(\mathbf{k}, \mathbf{J}, \omega)-i \mathbf{k} \cdot \frac{\partial f}{\partial \mathbf{J}} \delta \tilde{\Phi}(\mathbf{k}, \mathbf{J}, \omega)=0
$$

where the first term is the spatial Fourier transform of the initial value

$$
\delta \hat{f}(\mathbf{k}, \mathbf{J}, 0)=\int \frac{d \mathbf{w}}{(2 \pi)^{d}} e^{-i \mathbf{k} \cdot \mathbf{w}} \delta f(\mathbf{w}, \mathbf{J}, 0) .
$$


The foregoing equation can be rewritten

$$
\delta \tilde{f}(\mathbf{k}, \mathbf{J}, \omega)=\frac{\mathbf{k} \cdot \frac{\partial f}{\partial \mathbf{J}}}{\mathbf{k} \cdot \boldsymbol{\Omega}-\omega} \delta \tilde{\Phi}(\mathbf{k}, \mathbf{J}, \omega)+\frac{\delta \hat{f}(\mathbf{k}, \mathbf{J}, 0)}{i(\mathbf{k} \cdot \boldsymbol{\Omega}-\omega)},
$$

where the first term on the right hand side corresponds to "collective effects" and the second term is related to the initial condition. In order to simplify the notations, we have noted $\boldsymbol{\Omega}$ for $\boldsymbol{\Omega}(\mathbf{J})$. Similarly, we shall note $f$ for $f(\mathbf{J})$, $\boldsymbol{\Omega}^{\prime}$ for $\boldsymbol{\Omega}\left(\mathbf{J}^{\prime}\right)$ and $f^{\prime}$ for $f\left(\mathbf{J}^{\prime}\right)$. The fluctuations of the potential $\Phi(\mathbf{r}, t)$ are related to the fluctuations of the density $\rho(\mathbf{r}, t)=\int f(\mathbf{r}, \mathbf{v}, t) d \mathbf{v}$ by $\delta \Phi(\mathbf{r}, t)=\int u\left(\left|\mathbf{r}-\mathbf{r}^{\prime}\right|\right) \delta \rho\left(\mathbf{r}^{\prime}, t\right) d \mathbf{r}^{\prime}$. We introduce a bi-orthogonal basis [70] in which the density $\rho_{\alpha}(\mathbf{r})$ and the potential $\Phi_{\alpha}(\mathbf{r})$ are related to each other by

$$
\Phi_{\alpha}(\mathbf{r})=\int u\left(\left|\mathbf{r}-\mathbf{r}^{\prime}\right|\right) \rho_{\alpha}\left(\mathbf{r}^{\prime}\right) d \mathbf{r}^{\prime}, \quad \int \rho_{\alpha}(\mathbf{r}) \Phi_{\alpha^{\prime}}^{*}(\mathbf{r}) d \mathbf{r}=-\delta_{\alpha, \alpha^{\prime}}
$$

The fluctuations of density $\delta \rho(\mathbf{r}, t)$ and potential $\delta \Phi(\mathbf{r}, t)$ can be expanded on this basis as

$$
\delta \rho(\mathbf{r}, t)=\sum_{\alpha} A_{\alpha}(t) \rho_{\alpha}(\mathbf{r}), \quad \delta \Phi(\mathbf{r}, t)=\sum_{\alpha} A_{\alpha}(t) \Phi_{\alpha}(\mathbf{r}) .
$$

Introducing angle-action variables, we have

$$
\delta \Phi(\mathbf{w}, \mathbf{J}, t)=\sum_{\alpha} A_{\alpha}(t) \Phi_{\alpha}(\mathbf{w}, \mathbf{J}) .
$$

Taking the Fourier-Laplace transform of this equation, we obtain

$$
\delta \tilde{\Phi}(\mathbf{k}, \mathbf{J}, \omega)=\sum_{\alpha} \tilde{A}_{\alpha}(\omega) \hat{\Phi}_{\alpha}(\mathbf{k}, \mathbf{J})
$$

where

$$
\tilde{A}_{\alpha}(\omega)=\int_{0}^{+\infty} A_{\alpha}(t) e^{i \omega t} d t
$$

is the Laplace transform of $A_{\alpha}(t)$ and

$$
\hat{\Phi}_{\alpha}(\mathbf{k}, \mathbf{J})=\int \Phi_{\alpha}(\mathbf{w}, \mathbf{J}) e^{-i \mathbf{k} \cdot \mathbf{w}} \frac{d \mathbf{w}}{(2 \pi)^{d}},
$$

is the Fourier transform of $\Phi_{\alpha}(\mathbf{w}, \mathbf{J})$. We can rewrite Eq. (21) as

$$
\delta \tilde{f}(\mathbf{k}, \mathbf{J}, \omega)=\frac{\mathbf{k} \cdot \frac{\partial f}{\partial \mathbf{J}}}{\mathbf{k} \cdot \boldsymbol{\Omega}-\omega} \sum_{\alpha} \tilde{A}_{\alpha}(\omega) \hat{\Phi}_{\alpha}(\mathbf{k}, \mathbf{J})+\frac{\delta \hat{f}(\mathbf{k}, \mathbf{J}, 0)}{i(\mathbf{k} \cdot \mathbf{\Omega}-\omega)} .
$$

Taking the inverse Fourier transform of this expression, we obtain

$$
\delta \tilde{f}(\mathbf{w}, \mathbf{J}, \omega)=\sum_{\mathbf{k}} e^{i \mathbf{k} \cdot \mathbf{w}} \delta \tilde{f}(\mathbf{k}, \mathbf{J}, \omega)=\sum_{\mathbf{k}} e^{i \mathbf{k} \cdot \mathbf{w}}\left[\frac{\mathbf{k} \cdot \frac{\partial f}{\partial \mathbf{J}}}{\mathbf{k} \cdot \boldsymbol{\Omega}-\omega} \sum_{\alpha} \tilde{A}_{\alpha}(\omega) \hat{\Phi}_{\alpha}(\mathbf{k}, \mathbf{J})+\frac{\delta \hat{f}(\mathbf{k}, \mathbf{J}, 0)}{i(\mathbf{k} \cdot \boldsymbol{\Omega}-\omega)}\right] .
$$

Multiplying the left hand side of Eq. (29) by $\Phi_{\alpha^{\prime}}^{*}(\mathbf{w}, \mathbf{J})$ and integrating over $\mathbf{w}$ and $\mathbf{J}$, we get

$$
\begin{array}{r}
\int \delta \tilde{f}(\mathbf{w}, \mathbf{J}, \omega) \Phi_{\alpha^{\prime}}^{*}(\mathbf{w}, \mathbf{J}) d \mathbf{w} d \mathbf{J}=\int \delta \tilde{f}(\mathbf{r}, \mathbf{v}, \omega) \Phi_{\alpha^{\prime}}^{*}(\mathbf{r}) d \mathbf{r} d \mathbf{v}=\int \delta \tilde{\rho}(\mathbf{r}, \omega) \Phi_{\alpha^{\prime}}^{*}(\mathbf{r}) d \mathbf{r} \\
=\sum_{\alpha} \int \tilde{A}_{\alpha}(\omega) \rho_{\alpha}(\mathbf{r}) \Phi_{\alpha^{\prime}}^{*}(\mathbf{r}) d \mathbf{r}=-\sum_{\alpha} \tilde{A}_{\alpha}(\omega) \delta_{\alpha, \alpha^{\prime}}=-\tilde{A}_{\alpha^{\prime}}(\omega)
\end{array}
$$

where we have used the fact that $(\mathbf{w}, \mathbf{J})$ are canonical variables so that $d \mathbf{r} d \mathbf{v}=d \mathbf{w} d \mathbf{J}$. Multiplying the right hand side of Eq. (29) by $\Phi_{\alpha^{\prime}}^{*}(\mathbf{w}, \mathbf{J})$ and integrating over $\mathbf{w}$ and $\mathbf{J}$, we get

$$
\begin{aligned}
& \sum_{\mathbf{k}} \int d \mathbf{w} d \mathbf{J} e^{i \mathbf{k} \cdot \mathbf{w}} \Phi_{\alpha^{\prime}}^{*}(\mathbf{w}, \mathbf{J})\left[\frac{\mathbf{k} \cdot \frac{\partial f}{\partial \mathbf{J}}}{\mathbf{k} \cdot \boldsymbol{\Omega}-\omega} \sum_{\alpha} \tilde{A}_{\alpha}(\omega) \hat{\Phi}_{\alpha}(\mathbf{k}, \mathbf{J})+\frac{\delta \hat{f}(\mathbf{k}, \mathbf{J}, 0)}{i(\mathbf{k} \cdot \boldsymbol{\Omega}-\omega)}\right] \\
& =(2 \pi)^{d} \sum_{\mathbf{k}} \int d \mathbf{J} \hat{\Phi}_{\alpha^{\prime}}^{*}(\mathbf{k}, \mathbf{J})\left[\frac{\mathbf{k} \cdot \frac{\partial f}{\partial \mathbf{J}}}{\mathbf{k} \cdot \boldsymbol{\Omega}-\omega} \sum_{\alpha} \tilde{A}_{\alpha}(\omega) \hat{\Phi}_{\alpha}(\mathbf{k}, \mathbf{J})+\frac{\delta \hat{f}(\mathbf{k}, \mathbf{J}, 0)}{i(\mathbf{k} \cdot \boldsymbol{\Omega}-\omega)}\right] .
\end{aligned}
$$


From Eqs. (30) and (31), we obtain

$$
-\tilde{A}_{\alpha^{\prime}}(\omega)=(2 \pi)^{d} \sum_{\alpha}\left[\sum_{\mathbf{k}} \int d \mathbf{J} \frac{\mathbf{k} \cdot \frac{\partial f}{\partial \mathbf{J}}}{\mathbf{k} \cdot \boldsymbol{\Omega}-\omega} \hat{\Phi}_{\alpha}(\mathbf{k}, \mathbf{J}) \hat{\Phi}_{\alpha^{\prime}}^{*}(\mathbf{k}, \mathbf{J})\right] \tilde{A}_{\alpha}(\omega)+(2 \pi)^{d} \sum_{\mathbf{k}} \int d \mathbf{J} \frac{\delta \hat{f}(\mathbf{k}, \mathbf{J}, 0)}{i(\mathbf{k} \cdot \mathbf{\Omega}-\omega)} \hat{\Phi}_{\alpha^{\prime}}^{*}(\mathbf{k}, \mathbf{J}) .
$$

Introducing the dielectric tensor

$$
\epsilon_{\alpha \alpha^{\prime}}(\omega)=\delta_{\alpha \alpha^{\prime}}+(2 \pi)^{d} \sum_{\mathbf{k}} \int d \mathbf{J} \frac{\mathbf{k} \cdot \frac{\partial f}{\partial \mathbf{J}}}{\mathbf{k} \cdot \boldsymbol{\Omega}-\omega} \hat{\Phi}_{\alpha}^{*}(\mathbf{k}, \mathbf{J}) \hat{\Phi}_{\alpha^{\prime}}(\mathbf{k}, \mathbf{J})
$$

the foregoing relation can be rewritten [103]:

$$
\epsilon_{\alpha \alpha^{\prime}}(\omega) \tilde{A}_{\alpha^{\prime}}(\omega)=-(2 \pi)^{d} \sum_{\mathbf{k}} \int d \mathbf{J} \frac{\delta \hat{f}(\mathbf{k}, \mathbf{J}, 0)}{i(\mathbf{k} \cdot \mathbf{\Omega}-\omega)} \hat{\Phi}_{\alpha}^{*}(\mathbf{k}, \mathbf{J})
$$

Inverting the matrix $\epsilon(\omega)$, we get

$$
\tilde{A}_{\alpha}(\omega)=-(2 \pi)^{d} \sum_{\alpha^{\prime}}\left(\epsilon^{-1}\right)_{\alpha \alpha^{\prime}}(\omega) \sum_{\mathbf{k}^{\prime}} \int d \mathbf{J}^{\prime} \frac{\delta \hat{f}\left(\mathbf{k}^{\prime}, \mathbf{J}^{\prime}, 0\right)}{i\left(\mathbf{k}^{\prime} \cdot \mathbf{\Omega}^{\prime}-\omega\right)} \hat{\Phi}_{\alpha^{\prime}}^{*}\left(\mathbf{k}^{\prime}, \mathbf{J}^{\prime}\right) .
$$

Finally, substituting Eq. (35) in Eq. (25) and introducing the notation

$$
\frac{1}{D_{\mathbf{k}, \mathbf{k}^{\prime}}\left(\mathbf{J}, \mathbf{J}^{\prime}, \omega\right)}=\sum_{\alpha \alpha^{\prime}} \hat{\Phi}_{\alpha}(\mathbf{k}, \mathbf{J})\left(\epsilon^{-1}\right)_{\alpha \alpha^{\prime}}(\omega) \hat{\Phi}_{\alpha^{\prime}}^{*}\left(\mathbf{k}^{\prime}, \mathbf{J}^{\prime}\right)
$$

we obtain

$$
\delta \tilde{\Phi}(\mathbf{k}, \mathbf{J}, \omega)=-(2 \pi)^{d} \sum_{\mathbf{k}^{\prime}} \int d \mathbf{J}^{\prime} \frac{1}{D_{\mathbf{k}, \mathbf{k}^{\prime}}\left(\mathbf{J}, \mathbf{J}^{\prime}, \omega\right)} \frac{\delta \hat{f}\left(\mathbf{k}^{\prime}, \mathbf{J}^{\prime}, 0\right)}{i\left(\mathbf{k}^{\prime} \cdot \mathbf{\Omega}^{\prime}-\omega\right)}
$$

We can use the previous expressions to compute the collision term appearing on the right hand side of Eq. (15). One has

$$
\left\langle\delta f \frac{\partial \delta \Phi}{\partial \mathbf{w}}\right\rangle=\sum_{\mathbf{k}} \int_{\mathcal{C}} \frac{d \omega}{2 \pi} \sum_{\mathbf{k}^{\prime}} \int_{\mathcal{C}} \frac{d \omega^{\prime}}{2 \pi} i \mathbf{k}^{\prime} e^{i(\mathbf{k} \cdot \mathbf{w}-\omega t)} e^{i\left(\mathbf{k}^{\prime} \cdot \mathbf{w}-\omega^{\prime} t\right)}\left\langle\delta \tilde{f}(\mathbf{k}, \mathbf{J}, \omega) \delta \tilde{\Phi}\left(\mathbf{k}^{\prime}, \mathbf{J}, \omega^{\prime}\right)\right\rangle .
$$

Using Eq. (21), we find that

$$
\left\langle\delta \tilde{f}(\mathbf{k}, \mathbf{J}, \omega) \delta \tilde{\Phi}\left(\mathbf{k}^{\prime}, \mathbf{J}, \omega^{\prime}\right)\right\rangle=\frac{\mathbf{k} \cdot \frac{\partial f}{\partial \mathbf{J}}}{\mathbf{k} \cdot \boldsymbol{\Omega}-\omega}\left\langle\delta \tilde{\Phi}(\mathbf{k}, \mathbf{J}, \omega) \delta \tilde{\Phi}\left(\mathbf{k}^{\prime}, \mathbf{J}, \omega^{\prime}\right)\right\rangle+\frac{\left\langle\delta \tilde{f}(\mathbf{k}, \mathbf{J}, 0) \delta \tilde{\Phi}\left(\mathbf{k}^{\prime}, \mathbf{J}, \omega^{\prime}\right)\right\rangle}{i(\mathbf{k} \cdot \boldsymbol{\Omega}-\omega)} .
$$

The first term corresponds to the self-correlation of the potential, while the second term corresponds to the correlations between the fluctuations of the potential and of the distribution function at time $t=0$. Let us consider these two terms separately.

From Eq. (37), we obtain

$$
\left\langle\delta \tilde{\Phi}(\mathbf{k}, \mathbf{J}, \omega) \delta \tilde{\Phi}\left(\mathbf{k}^{\prime}, \mathbf{J}, \omega^{\prime}\right)\right\rangle=-(2 \pi)^{2 d} \sum_{\mathbf{k}_{1}, \mathbf{k}_{2}} \int d \mathbf{J}_{1} d \mathbf{J}_{2} \frac{1}{D_{\mathbf{k}, \mathbf{k}_{1}}\left(\mathbf{J}, \mathbf{J}_{1}, \omega\right)} \frac{1}{D_{\mathbf{k}^{\prime}, \mathbf{k}_{2}}\left(\mathbf{J}, \mathbf{J}_{2}, \omega^{\prime}\right)} \frac{\left\langle\delta \hat{f}\left(\mathbf{k}_{1}, \mathbf{J}_{1}, 0\right) \delta \hat{f}\left(\mathbf{k}_{2}, \mathbf{J}_{2}, 0\right)\right\rangle}{\left(\mathbf{k}_{1} \cdot \mathbf{\Omega}_{1}-\omega\right)\left(\mathbf{k}_{2} \cdot \mathbf{\Omega}_{2}-\omega^{\prime}\right)} .
$$

Using the expression of the auto-correlation of the fluctuations at $t=0$ given by (see Appendix C):

$$
\left\langle\delta \hat{f}(\mathbf{k}, \mathbf{J}, 0) \delta \hat{f}\left(\mathbf{k}^{\prime}, \mathbf{J}^{\prime}, 0\right)\right\rangle=\frac{1}{(2 \pi)^{d}} \delta_{\mathbf{k},-\mathbf{k}^{\prime}} \delta\left(\mathbf{J}-\mathbf{J}^{\prime}\right) m f(\mathbf{J}),
$$

we find that

$$
\left\langle\delta \tilde{\Phi}(\mathbf{k}, \mathbf{J}, \omega) \delta \tilde{\Phi}\left(\mathbf{k}^{\prime}, \mathbf{J}, \omega^{\prime}\right)\right\rangle=(2 \pi)^{d} \sum_{\mathbf{k}_{1}} \int d \mathbf{J}_{1} \frac{1}{D_{\mathbf{k}, \mathbf{k}_{1}}\left(\mathbf{J}, \mathbf{J}_{1}, \omega\right)} \frac{1}{D_{\mathbf{k}^{\prime},-\mathbf{k}_{1}}\left(\mathbf{J}, \mathbf{J}_{1}, \omega^{\prime}\right)} \frac{m f\left(\mathbf{J}_{1}\right)}{\left(\mathbf{k}_{1} \cdot \boldsymbol{\Omega}_{1}-\omega\right)\left(\mathbf{k}_{1} \cdot \mathbf{\Omega}_{1}+\omega^{\prime}\right)}
$$


Considering only the contributions that do not decay in time, it can be shown $[72]$ that $\left[\left(\mathbf{k}_{1} \cdot \boldsymbol{\Omega}_{1}-\omega\right)\left(\mathbf{k}_{1} \cdot \boldsymbol{\Omega}_{1}+\omega^{\prime}\right)\right]^{-1}$ can be substituted by $(2 \pi)^{2} \delta\left(\omega+\omega^{\prime}\right) \delta\left(\mathbf{k}_{1} \cdot \boldsymbol{\Omega}_{1}-\omega\right)$. Therefore

$$
\left\langle\delta \tilde{\Phi}(\mathbf{k}, \mathbf{J}, \omega) \delta \tilde{\Phi}\left(\mathbf{k}^{\prime}, \mathbf{J}, \omega^{\prime}\right)\right\rangle=(2 \pi)^{d+2} m \delta\left(\omega+\omega^{\prime}\right) \sum_{\mathbf{k}_{1}} \int d \mathbf{J}_{1} \frac{1}{D_{\mathbf{k}, \mathbf{k}_{1}}\left(\mathbf{J}, \mathbf{J}_{1}, \omega\right)} \frac{1}{D_{\mathbf{k}^{\prime},-\mathbf{k}_{1}}\left(\mathbf{J}, \mathbf{J}_{1},-\omega\right)} \delta\left(\mathbf{k}_{1} \cdot \mathbf{\Omega}_{1}-\omega\right) f\left(\mathbf{J}_{1}\right) .
$$

Similarly, one finds that the second term on the right hand side of Eq. (39) is given by

$$
\frac{\left\langle\delta \hat{f}(\mathbf{k}, \mathbf{J}, 0) \delta \tilde{\Phi}\left(\mathbf{k}^{\prime}, \mathbf{J}, \omega^{\prime}\right)\right\rangle}{i(\mathbf{k} \cdot \boldsymbol{\Omega}-\omega)}=-(2 \pi)^{2} m \delta\left(\omega+\omega^{\prime}\right) \frac{1}{D_{\mathbf{k}^{\prime},-\mathbf{k}}(\mathbf{J}, \mathbf{J},-\omega)} \delta(\mathbf{k} \cdot \boldsymbol{\Omega}-\omega) f(\mathbf{J}) .
$$

From Eq. (43), we get the contribution to Eq. (38) of the first term of Eq. (39). Since the collision term depends only on the action, it can be averaged over $\mathbf{w}$ without loss of information. This brings a Kronecker factor $\delta_{\mathbf{k},-\mathbf{k}^{\prime}}$ which amounts to taking $\mathbf{k}^{\prime}=-\mathbf{k}$. Then, using $D_{-\mathbf{k},-\mathbf{k}_{1}}\left(\mathbf{J}, \mathbf{J}_{1},-\omega\right)=D_{\mathbf{k}, \mathbf{k}_{1}}\left(\mathbf{J}, \mathbf{J}_{1}, \omega\right)^{*}$ [104], we obtain

$$
\left\langle\delta f \frac{\partial \delta \Phi}{\partial \mathbf{w}}\right\rangle_{I}=-i(2 \pi)^{d+1} m \sum_{\mathbf{k}, \mathbf{k}^{\prime}} \int_{\mathcal{C}} \frac{d \omega}{2 \pi} \int d \mathbf{J}^{\prime} \mathbf{k} \frac{\mathbf{k} \cdot \frac{\partial f}{\partial \mathbf{J}}}{\mathbf{k} \cdot \boldsymbol{\Omega}-\omega} \frac{1}{\left|D_{\mathbf{k}, \mathbf{k}^{\prime}}\left(\mathbf{J}, \mathbf{J}^{\prime}, \omega\right)\right|^{2}} \delta\left(\mathbf{k}^{\prime} \cdot \boldsymbol{\Omega}^{\prime}-\omega\right) f\left(\mathbf{J}^{\prime}\right) .
$$

Finally, using the Landau prescription $\omega \rightarrow \omega+i 0^{+}$and the Plemelj formula

$$
\frac{1}{x \pm i 0^{+}}=\mathcal{P}\left(\frac{1}{x}\right) \mp i \pi \delta(x)
$$

where $\mathcal{P}$ denotes the principal value, we can replace $1 /\left(\mathbf{k} \cdot \boldsymbol{\Omega}-\omega-i 0^{+}\right)$by $+i \pi \delta(\mathbf{k} \cdot \boldsymbol{\Omega}-\omega)$. Then, integrating over $\omega$, we obtain

$$
\left\langle\delta f \frac{\partial \delta \Phi}{\partial \mathbf{w}}\right\rangle_{I}=\pi(2 \pi)^{d} m \sum_{\mathbf{k}, \mathbf{k}^{\prime}} \int d \mathbf{J}^{\prime} \mathbf{k} \frac{1}{\left|D_{\mathbf{k}, \mathbf{k}^{\prime}}\left(\mathbf{J}, \mathbf{J}^{\prime}, \mathbf{k} \cdot \boldsymbol{\Omega}\right)\right|^{2}} \delta\left(\mathbf{k} \cdot \boldsymbol{\Omega}-\mathbf{k}^{\prime} \cdot \boldsymbol{\Omega}^{\prime}\right)\left(\mathbf{k} \cdot \frac{\partial f}{\partial \mathbf{J}}\right) f\left(\mathbf{J}^{\prime}\right) .
$$

From Eq. (44), we get the contribution to Eq. (38) of the second term of Eq. (39). It is given by

$$
\left\langle\delta f \frac{\partial \delta \Phi}{\partial \mathbf{w}}\right\rangle_{I I}=\sum_{\mathbf{k}} \mathbf{k} \operatorname{Im}\left(\frac{1}{D_{\mathbf{k}, \mathbf{k}}(\mathbf{J}, \mathbf{J}, \mathbf{k} \cdot \boldsymbol{\Omega})}\right) m f(\mathbf{J}) .
$$

Using Eq. (36) and introducing the convention of summation over repeated indices, we have

$$
\frac{1}{D_{\mathbf{k}, \mathbf{k}}(\mathbf{J}, \mathbf{J}, \mathbf{k} \cdot \boldsymbol{\Omega})}=\hat{\Phi}_{\alpha}(\mathbf{k}, \mathbf{J})\left(\epsilon^{-1}\right)_{\alpha \alpha^{\prime}}(\mathbf{k} \cdot \boldsymbol{\Omega}) \hat{\Phi}_{\alpha^{\prime}}^{*}(\mathbf{k}, \mathbf{J}) .
$$

Therefore

$$
\operatorname{Im}\left(\frac{1}{D_{\mathbf{k}, \mathbf{k}}(\mathbf{J}, \mathbf{J}, \mathbf{k} \cdot \boldsymbol{\Omega})}\right)=\frac{1}{2 i} \hat{\Phi}_{\alpha}(\mathbf{k}, \mathbf{J})\left[\left(\epsilon^{-1}\right)_{\alpha \alpha^{\prime}}(\mathbf{k} \cdot \boldsymbol{\Omega})-\left(\left(\epsilon^{-1}\right)_{\alpha^{\prime} \alpha}(\mathbf{k} \cdot \boldsymbol{\Omega})\right)^{*}\right] \hat{\Phi}_{\alpha^{\prime}}^{*}(\mathbf{k}, \mathbf{J}) .
$$

To compute the term in brackets, we use the identity

$$
\left(\epsilon^{-1}\right)-\left(\epsilon^{-1}\right)^{\dagger}=\epsilon^{-1}\left(\epsilon^{\dagger}-\epsilon\right)\left(\epsilon^{\dagger}\right)^{-1} .
$$

In component form, it can be written

$$
\left(\epsilon^{-1}\right)_{\alpha \alpha^{\prime}}-\left(\left(\epsilon^{-1}\right)_{\alpha^{\prime} \alpha}\right)^{*}=\left(\epsilon^{-1}\right)_{\alpha \lambda}\left(\epsilon_{\lambda^{\prime} \lambda}^{*}-\epsilon_{\lambda \lambda^{\prime}}\right)\left(\left(\epsilon^{-1}\right)_{\alpha^{\prime} \lambda^{\prime}}\right)^{*}
$$

From the expression (33) of the dielectric tensor, we find that

$$
\epsilon_{\lambda^{\prime} \lambda}(\omega)^{*}-\epsilon_{\lambda \lambda^{\prime}}(\omega)=-i(2 \pi)^{d+1} \sum_{\mathbf{k}} \int d \mathbf{J}\left(\mathbf{k} \cdot \frac{\partial f}{\partial \mathbf{J}}\right) \delta(\mathbf{k} \cdot \boldsymbol{\Omega}-\omega) \hat{\Phi}_{\lambda}^{*}(\mathbf{k}, \mathbf{J}) \hat{\Phi}_{\lambda^{\prime}}(\mathbf{k}, \mathbf{J}),
$$

where we have used the Landau prescription $\omega \rightarrow \omega+i 0^{+}$and the Plemelj formula (46). Inserting Eqs. (52) and (53) in Eq. (50) and using Eq. (36), we obtain

$$
\operatorname{Im}\left(\frac{1}{D_{\mathbf{k}, \mathbf{k}}(\mathbf{J}, \mathbf{J}, \mathbf{k} \cdot \boldsymbol{\Omega})}\right)=-\pi(2 \pi)^{d} \sum_{\mathbf{k}^{\prime}} \int d \mathbf{J}^{\prime} \frac{1}{\left|D_{\mathbf{k}, \mathbf{k}^{\prime}}\left(\mathbf{J}, \mathbf{J}^{\prime}, \mathbf{k} \cdot \boldsymbol{\Omega}\right)\right|^{2}} \delta\left(\mathbf{k} \cdot \boldsymbol{\Omega}-\mathbf{k}^{\prime} \cdot \mathbf{\Omega}^{\prime}\right)\left(\mathbf{k}^{\prime} \cdot \frac{\partial f^{\prime}}{\partial \mathbf{J}^{\prime}}\right) .
$$


Substituting Eq. (54) in Eq. (48), we find that

$$
\left\langle\delta f \frac{\partial \delta \Phi}{\partial \mathbf{w}}\right\rangle_{I I}=-\pi(2 \pi)^{d} m \sum_{\mathbf{k}, \mathbf{k}^{\prime}} \int d \mathbf{J}^{\prime} \mathbf{k} \frac{1}{\left|D_{\mathbf{k}, \mathbf{k}^{\prime}}\left(\mathbf{J}, \mathbf{J}^{\prime}, \mathbf{k} \cdot \boldsymbol{\Omega}\right)\right|^{2}} \delta\left(\mathbf{k} \cdot \boldsymbol{\Omega}-\mathbf{k}^{\prime} \cdot \boldsymbol{\Omega}^{\prime}\right)\left(\mathbf{k}^{\prime} \cdot \frac{\partial f^{\prime}}{\partial \mathbf{J}^{\prime}}\right) f(\mathbf{J}) .
$$

Finally, regrouping Eqs. (15), (47) and (55) and restoring the time variable, we end up with the kinetic equation

$$
\frac{\partial f}{\partial t}=\pi(2 \pi)^{d} m \frac{\partial}{\partial \mathbf{J}} \cdot \sum_{\mathbf{k}, \mathbf{k}^{\prime}} \int d \mathbf{J}^{\prime} \mathbf{k} \frac{1}{\left|D_{\mathbf{k}, \mathbf{k}^{\prime}}\left(\mathbf{J}, \mathbf{J}^{\prime}, \mathbf{k} \cdot \boldsymbol{\Omega}\right)\right|^{2}} \delta\left(\mathbf{k} \cdot \boldsymbol{\Omega}-\mathbf{k}^{\prime} \cdot \boldsymbol{\Omega}^{\prime}\right)\left(\mathbf{k} \cdot \frac{\partial}{\partial \mathbf{J}}-\mathbf{k}^{\prime} \cdot \frac{\partial}{\partial \mathbf{J}^{\prime}}\right) f(\mathbf{J}, t) f\left(\mathbf{J}^{\prime}, t\right) .
$$

This Lenard-Balescu-type kinetic equation, taking spatial inhomogeneity and collective effects into account, was derived by Heyvaerts [69] from the BBGKY hierarchy. It describes the "collisional" evolution of the distribution function caused by the weak noise created by the discreteness of the particles accompanied by their associated polarization cloud. It is written in action space, which is possible when the Hamiltonian associated with the average potential is integrable. For spatially homogeneous systems, it reduces to the ordinary Lenard-Balescu equation

$$
\frac{\partial f}{\partial t}=\pi(2 \pi)^{d} m \frac{\partial}{\partial \mathbf{v}} \cdot \int d \mathbf{k} d \mathbf{v}^{\prime} \mathbf{k} \frac{\hat{u}(\mathbf{k})^{2}}{|\epsilon(\mathbf{k}, \mathbf{k} \cdot \mathbf{v})|^{2}} \delta\left[\mathbf{k} \cdot\left(\mathbf{v}-\mathbf{v}^{\prime}\right)\right] \mathbf{k} \cdot\left(\frac{\partial}{\partial \mathbf{v}}-\frac{\partial}{\partial \mathbf{v}^{\prime}}\right) f(\mathbf{v}, t) f\left(\mathbf{v}^{\prime}, t\right)
$$

where

$$
\epsilon(\mathbf{k}, \omega)=1+(2 \pi)^{d} \hat{u}(\mathbf{k}) \int \frac{\mathbf{k} \cdot \frac{\partial f}{\partial \mathbf{v}}}{\omega-\mathbf{k} \cdot \mathbf{v}} d \mathbf{v},
$$

is the dielectric function. When collective effects are neglected, we obtained in Refs. [67, 68] a Landau-type kinetic equation with angle-action variables of the form

$$
\frac{\partial f}{\partial t}=\pi(2 \pi)^{d} m \frac{\partial}{\partial \mathbf{J}} \cdot \sum_{\mathbf{k}, \mathbf{k}^{\prime}} \int d \mathbf{J}^{\prime} \mathbf{k}\left|A_{\mathbf{k}, \mathbf{k}^{\prime}}\left(\mathbf{J}, \mathbf{J}^{\prime}\right)\right|^{2} \delta\left(\mathbf{k} \cdot \boldsymbol{\Omega}-\mathbf{k}^{\prime} \cdot \boldsymbol{\Omega}^{\prime}\right)\left(\mathbf{k} \cdot \frac{\partial}{\partial \mathbf{J}}-\mathbf{k}^{\prime} \cdot \frac{\partial}{\partial \mathbf{J}^{\prime}}\right) f(\mathbf{J}, t) f\left(\mathbf{J}^{\prime}, t\right),
$$

where $A_{\mathbf{k}, \mathbf{k}^{\prime}}\left(\mathbf{J}, \mathbf{J}^{\prime}\right)$ is the Fourier transform of the potential of interaction written in angle-action variables (see Appendix A . This equation was derived in [68] by a method that does not use Fourier-Laplace transforms nor biorthogonal basis. It arises from the generalized Landau equation

$$
\begin{aligned}
& \frac{\partial f}{\partial t}+\mathbf{v} \cdot \frac{\partial f}{\partial \mathbf{r}}+\frac{N-1}{N}\langle\mathbf{F}\rangle \cdot \frac{\partial f}{\partial \mathbf{v}}=\frac{\partial}{\partial v^{\mu}} \int_{0}^{t} d \tau \int d \mathbf{r}_{1} d \mathbf{v}_{1} F^{\mu}(1 \rightarrow 0) \mathcal{G}(t, t-\tau) \\
& \times\left[\mathcal{F}^{\nu}(1 \rightarrow 0) \frac{\partial}{\partial v^{\nu}}+\mathcal{F}^{\nu}(0 \rightarrow 1) \frac{\partial}{\partial v_{1}^{\nu}}\right] f(\mathbf{r}, \mathbf{v}, t-\tau) \frac{f}{m}\left(\mathbf{r}_{1}, \mathbf{v}_{1}, t-\tau\right),
\end{aligned}
$$

derived in 35 37], which is valid for systems that are not necessarily spatially homogeneous and not necessarily Markovian [105]. The Landau-type equation (59) is obtained from Eq. (60) by making a Markovian approximation and introducing angle-action variables [68]. For spatially homogeneous systems, it reduces to the ordinary Landau equation corresponding to Eq. (57) with $|\epsilon(\mathbf{k}, \mathbf{k} \cdot \mathbf{v})|=1$. The connection between Eqs. (56) and (59) is clear. If we neglect collective effects, $1 / D_{\mathbf{k}, \mathbf{k}^{\prime}}\left(\mathbf{J}, \mathbf{J}^{\prime}, \omega\right)_{\text {bare }}$ is just the Fourier transform $A_{\mathbf{k}, \mathbf{k}^{\prime}}\left(\mathbf{J}, \mathbf{J}^{\prime}\right)$ of the "bare" potential of interaction (see Appendix A). When collective effects are taken into account, the "bare" potential of interaction $A_{\mathbf{k}, \mathbf{k}^{\prime}}\left(\mathbf{J}, \mathbf{J}^{\prime}\right)$ is replaced by the "dressed" potential of interaction $1 / D_{\mathbf{k}, \mathbf{k}^{\prime}}\left(\mathbf{J}, \mathbf{J}^{\prime}, \omega\right)$, without changing the overall structure of the kinetic equation. This is similar to the case of homogeneous plasmas where the bare potential $\hat{u}(\mathbf{k})$ in the Landau equation is replaced by the "dressed" potential $\hat{u}(\mathbf{k}) /|\epsilon(\mathbf{k}, \mathbf{k} \cdot \mathbf{v})|$ in the Lenard-Balescu equation.

\section{The relaxation time of the system as a whole}

The kinetic equation (56) is valid at the order $1 / N$ so it describes the "collisional" evolution of the system on a timescale $\sim N t_{D}$. This kinetic equation conserves the total mass $M=\int f(\mathbf{J}, t) d \mathbf{J}$ and the total energy $E=$ $\int f(\mathbf{J}, t) H(\mathbf{J}) d \mathbf{J}$. It also monotonically increases the Boltzmann entropy $S=-\int(f / m)(\mathbf{J}, t) \ln (f / m)(\mathbf{J}, t) d \mathbf{J}(H-$ theorem). The Boltzmann distribution $f_{e}(\mathbf{J})=A e^{-\beta m H(\mathbf{J})}$ is a steady state of this kinetic equation. The derivation of these results can be found in [67, 69]. The collisional evolution of the system (at the order $1 / N$ ) is due to a condition of resonance between distant orbits. The condition of resonance, encapsulated in the $\delta$-function, corresponds to 
$\mathbf{k}^{\prime} \cdot \boldsymbol{\Omega}\left(\mathbf{J}^{\prime}\right)=\mathbf{k} \cdot \boldsymbol{\Omega}(\mathbf{J})$ with $\left(\mathbf{k}^{\prime}, \mathbf{J}^{\prime}\right) \neq(\mathbf{k}, \mathbf{J})$. If there are resonances during the whole evolution, the system will relax towards the Boltzmann distribution (provided that this equilibrium state exists). In that case, the relaxation time is

$$
t_{R} \sim N t_{D} .
$$

This is the case, in general, for spatially inhomogeneous systems in any dimension [106]. This is also the case for spatially homogeneous systems in $d>1$ dimensions. In that case, the condition of resonance corresponds to $\mathbf{k} \cdot \mathbf{v}^{\prime}=\mathbf{k} \cdot \mathbf{v}$ with $\mathbf{v}^{\prime} \neq \mathbf{v}$ (the self-interaction at $\mathbf{v}=\mathbf{v}^{\prime}$ does not produce transport since the term in parenthesis in Eq. (57) vanishes identically). However, for spatially homogeneous one dimensional systems, there is no resonance and the Lenard-Balescu collision term vanishes [68, 80]. The kinetic equation (57) reduces to $\partial f / \partial t=0$, so the distribution function does not evolve at all on a timescale $\sim N t_{D}$. In that case, the relaxation time is larger than $N t_{D}$. We therefore expect that

$$
t_{R}>N t_{D} \quad \text { (1D homogeneous). }
$$

Since the relaxation process is due to more complex correlations, we have to develop the kinetic theory at higher orders (taking three-body, four-body,... correlation functions into account) to obtain the relaxation time. If the collision term does not vanish at the next order of the expansion in powers of $1 / N$, the kinetic theory would imply a relaxation time of the order $N^{2} t_{D}$. However, the problem could be more complicated and yield a larger relaxation time (see the Conclusion for a more detailed discussion). In fact, it is not even granted that the system will ever relax towards statistical equilibrium. The evolution may be non-ergodic and the mixing by "collisions" inefficient. By contrast, for spatially homogeneous systems in $d>1$ dimensions [see Eq. [57]], and for spatially inhomogeneous systems in any dimension [see Eq. (56)], since there are potentially more resonances, the relaxation time could be reduced and approach the natural scaling $N t_{D}$ predicted by the first order kinetic theory [67, 68]. However, very little is known concerning the properties of Eq. (56) and its convergence (or not) towards the Boltzmann distribution. It could approach the Boltzmann distribution (since entropy increases), without reaching it exactly if the resonances disappear at some point of the evolution (see Refs. [67, 68] for more details).

\section{STOCHASTIC PROCESS OF A TEST PARTICLE: DIFFUSION AND FRICTION}

\section{A. The Fokker-Planck equation}

In the previous section, we have studied the evolution of the system as a whole. We now consider the relaxation of a test particle is a bath of field particles with a steady distribution $f(\mathbf{J})$. We assume that the field particles are either at statistical equilibrium with the Boltzmann distribution (thermal bath), in which case their distribution does not change at all, or in a stable steady state of the Vlasov equation with a profile that forbids any resonance (as we have just seen, this profile does not change on a timescale of order $N t_{D}$ ). We assume that the test particle has an initial action $\mathbf{J}_{0}$ and we study how it progressively acquires the distribution of the bath due to "collisions" with the field particles. As we shall see, the test particle has a stochastic motion and the evolution of the distribution function $P(\mathbf{J}, t)$, the probability density that the test particle has an action $\mathbf{J}$ at time $t$, is governed by a Fokker-Planck equation involving a diffusion term and a friction term that can be analytically obtained. The Fokker-Planck equation may then be solved with the initial condition $P(\mathbf{J}, 0)=\delta\left(\mathbf{J}-\mathbf{J}_{0}\right)$ to yield $P(\mathbf{J}, t)$. This problem has been investigated in our previous papers [67, 68], but we shall give here a direct and more rigorous derivation of the coefficients of diffusion and friction, taking collective effects into account.

The equations of motion of the test particle, written with angle-action variables, are

$$
\frac{d \mathbf{w}}{d t}=\boldsymbol{\Omega}(\mathbf{J})+\frac{\partial \delta \Phi}{\partial \mathbf{J}}(\mathbf{w}, \mathbf{J}, t), \quad \frac{d \mathbf{J}}{d t}=-\frac{\partial \delta \Phi}{\partial \mathbf{w}}(\mathbf{w}, \mathbf{J}, t) .
$$

They include the effect of the mean field which produces a zeroth order motion characterized by the pulsation $\boldsymbol{\Omega}(\mathbf{J})$ plus a stochastic component $\delta \Phi$ of order $1 / \sqrt{N}$ (fluctuations) which takes the deviations from the mean field into account. They can be formally integrated into

$$
\begin{gathered}
\mathbf{w}(t)=\mathbf{w}+\int_{0}^{t} \boldsymbol{\Omega}\left(\mathbf{J}\left(t^{\prime}\right)\right) d t^{\prime}+\int_{0}^{t} \frac{\partial \delta \Phi}{\partial \mathbf{J}}\left(\mathbf{w}\left(t^{\prime}\right), \mathbf{J}\left(t^{\prime}\right), t^{\prime}\right) d t^{\prime}, \\
\mathbf{J}(t)=\mathbf{J}-\int_{0}^{t} \frac{\partial \delta \Phi}{\partial \mathbf{w}}\left(\mathbf{w}\left(t^{\prime}\right), \mathbf{J}\left(t^{\prime}\right), t^{\prime}\right) d t^{\prime},
\end{gathered}
$$


where we have assumed that, initially, the test particle is at $(\mathbf{w}, \mathbf{J})$. Note that the "initial" time considered here does not necessarily coincide with the original time mentioned above. Since the fluctuation $\delta \Phi$ of the potential is a small quantity, the foregoing equations can be solved iteratively. At the order $1 / N$, which corresponds to quadratic order in $\delta \Phi$, we get for the action

$$
\begin{aligned}
J_{i}(t)=J_{i} & -\int_{0}^{t} d t^{\prime} \frac{\partial \delta \Phi}{\partial w_{i}}\left(\mathbf{w}+\boldsymbol{\Omega} t^{\prime}, \mathbf{J}, t^{\prime}\right)+\frac{\partial \Omega_{l}}{\partial J_{j}} \int_{0}^{t} d t^{\prime} \int_{0}^{t^{\prime}} d t^{\prime \prime} \int_{0}^{t^{\prime \prime}} d t^{\prime \prime \prime} \frac{\partial^{2} \delta \Phi}{\partial w_{i} \partial w_{l}}\left(\mathbf{w}+\boldsymbol{\Omega} t^{\prime}, \mathbf{J}, t^{\prime}\right) \frac{\partial \delta \Phi}{\partial w_{j}}\left(\mathbf{w}+\boldsymbol{\Omega} t^{\prime \prime \prime}, \mathbf{J}, t^{\prime \prime \prime}\right) \\
& +\int_{0}^{t} d t^{\prime} \int_{0}^{t^{\prime}} d t^{\prime \prime} \frac{\partial^{2} \delta \Phi}{\partial w_{i} \partial J_{j}}\left(\mathbf{w}+\boldsymbol{\Omega} t^{\prime}, \mathbf{J}, t^{\prime}\right) \frac{\partial \delta \Phi}{\partial w_{j}}\left(\mathbf{w}+\boldsymbol{\Omega} t^{\prime \prime}, \mathbf{J}, t^{\prime \prime}\right) \\
& -\int_{0}^{t} d t^{\prime} \int_{0}^{t^{\prime}} d t^{\prime \prime} \frac{\partial^{2} \delta \Phi}{\partial w_{i} \partial w_{j}}\left(\mathbf{w}+\boldsymbol{\Omega} t^{\prime}, \mathbf{J}, t^{\prime}\right) \frac{\partial \delta \Phi}{\partial J_{j}}\left(\mathbf{w}+\boldsymbol{\Omega} t^{\prime \prime}, \mathbf{J}, t^{\prime \prime}\right) .
\end{aligned}
$$

As the changes in the action are small, the dynamics of the test particle can be represented by a stochastic process governed by a Fokker-Planck equation [81]. If we denote by $P(\mathbf{J}, t)$ the probability density that the test particle has an action $\mathbf{J}$ at time $t$, the general form of this equation is

$$
\frac{\partial P}{\partial t}=\frac{\partial^{2}}{\partial J_{i} \partial J_{j}}\left(D_{i j} P\right)-\frac{\partial}{\partial J_{i}}\left(P A_{i}\right) .
$$

The diffusion tensor and the friction force are defined by

$$
\begin{gathered}
D_{i j}(\mathbf{J})=\lim _{t \rightarrow+\infty} \frac{1}{2 t}\left\langle\left(J_{i}(t)-J_{i}\right)\left(J_{j}(t)-J_{j}\right)\right\rangle, \\
A_{i}(\mathbf{J})=\lim _{t \rightarrow+\infty} \frac{1}{t}\left\langle J_{i}(t)-J_{i}\right\rangle .
\end{gathered}
$$

In writing these limits, we have implicitly assumed that the time $t$ is long with respect to the fluctuation time but short with respect to the relaxation time (of order $N t_{D}$ ), so the expression (66) can be used to evaluate Eqs. (68) and (69). As shown in our previous papers [67, 68], it is relevant to rewrite the Fokker-Planck equation in the alternative form

$$
\frac{\partial P}{\partial t}=\frac{\partial}{\partial J_{i}}\left(D_{i j} \frac{\partial P}{\partial J_{j}}-P F_{i}^{p o l}\right)
$$

The total friction is

$$
A_{i}=F_{i}^{p o l}+\frac{\partial D_{i j}}{\partial J_{j}}
$$

where $\mathbf{F}_{p o l}$ is the friction due to the polarization, while the second term is due to the variations of the diffusion tensor with $\mathbf{J}$. As we shall see, this decomposition arises naturally in the following analysis. The two expressions (67) and (70) have their own interest. The expression (67) where the diffusion tensor is placed after the second derivative $\partial^{2}(D P)$ involves the total friction $\mathbf{A}$ and the expression (70) where the diffusion tensor is placed between the derivatives $\partial D \partial P$ isolates the friction by polarization $\mathbf{F}_{p o l}$. We shall see in Sec. IIIE that this second form is directly related to the Lenard-Balescu-type equation (56). It has therefore a clear physical interpretation. We now calculate the diffusion tensor and the friction force from Eqs. (68) and (69), using the results of Sec. IC that allow to take collective effects into account. The reader not interested in the details of the calculations may directly go to the final results summarized in Sec. IIIE

\section{B. The diffusion tensor}

We first compute the diffusion tensor defined by Eq. (68). Using Eq. (66), we see that it is given, at the order $1 / N$, by

$$
D_{i j}=\frac{1}{2 t} \int_{0}^{t} d t^{\prime} \int_{0}^{t} d t^{\prime \prime}\left\langle\frac{\partial \delta \Phi}{\partial w_{i}}\left(\mathbf{w}+\boldsymbol{\Omega} t^{\prime}, \mathbf{J}, t^{\prime}\right) \frac{\partial \delta \Phi}{\partial w_{j}}\left(\mathbf{w}+\boldsymbol{\Omega} t^{\prime \prime}, \mathbf{J}, t^{\prime \prime}\right)\right\rangle
$$


By the inverse Fourier-Laplace transform, we have

$$
\begin{array}{r}
\left\langle\frac{\partial \delta \Phi}{\partial w_{i}}\left(\mathbf{w}+\boldsymbol{\Omega} t^{\prime}, \mathbf{J}, t^{\prime}\right) \frac{\partial \delta \Phi}{\partial w_{j}}\left(\mathbf{w}+\boldsymbol{\Omega} t^{\prime \prime}, \mathbf{J}, t^{\prime \prime}\right)\right\rangle=-\sum_{\mathbf{k}} \int_{\mathcal{C}} \frac{d \omega}{2 \pi} \sum_{\mathbf{k}^{\prime}} \int_{\mathcal{C}} \frac{d \omega^{\prime}}{2 \pi} k_{i} k_{j}^{\prime} e^{i \mathbf{k} \cdot\left(\mathbf{w}+\boldsymbol{\Omega} t^{\prime}\right)} e^{-i \omega t^{\prime}} e^{i \mathbf{k}^{\prime} \cdot\left(\mathbf{w}+\boldsymbol{\Omega} t^{\prime \prime}\right)} e^{-i \omega^{\prime} t^{\prime \prime}} \\
\times\left\langle\delta \tilde{\Phi}(\mathbf{k}, \mathbf{J}, \omega) \delta \tilde{\Phi}\left(\mathbf{k}^{\prime}, \mathbf{J}, \omega^{\prime}\right)\right\rangle .
\end{array}
$$

Since the diffusion tensor depends only on the action, it can be averaged over w without loss of information. This brings a Kronecker factor $\delta_{\mathbf{k},-\mathbf{k}^{\prime}}$ which amounts to taking $\mathbf{k}^{\prime}=-\mathbf{k}$. Then, substituting Eq. (43) in Eq. (73), and carrying out the integrals over $\omega^{\prime}$ and $\omega$, we end up with the result

$$
\left\langle\frac{\partial \delta \Phi}{\partial w_{i}}\left(\mathbf{w}+\boldsymbol{\Omega} t^{\prime}, \mathbf{J}, t^{\prime}\right) \frac{\partial \delta \Phi}{\partial w_{j}}\left(\mathbf{w}+\boldsymbol{\Omega} t^{\prime \prime}, \mathbf{J}, t^{\prime \prime}\right)\right\rangle=(2 \pi)^{d} m \sum_{\mathbf{k}, \mathbf{k}^{\prime}} \int d \mathbf{J}^{\prime} k_{i} k_{j} e^{i\left(\mathbf{k} \cdot \boldsymbol{\Omega}-\mathbf{k}^{\prime} \cdot \mathbf{\Omega}^{\prime}\right)\left(t^{\prime}-t^{\prime \prime}\right)} \frac{1}{\left|D_{\mathbf{k}, \mathbf{k}^{\prime}}\left(\mathbf{J}, \mathbf{J}^{\prime}, \mathbf{k}^{\prime} \cdot \mathbf{\Omega}^{\prime}\right)\right|^{2}} f\left(\mathbf{J}^{\prime}\right) .
$$

This expression shows that the correlation function appearing in Eq. (72) is an even function of $t^{\prime}-t^{\prime \prime}$. Using the identity

$$
\int_{0}^{t} d t^{\prime} \int_{0}^{t} d t^{\prime \prime} f\left(t^{\prime}-t^{\prime \prime}\right)=2 \int_{0}^{t} d t^{\prime} \int_{0}^{t^{\prime}} d t^{\prime \prime} f\left(t^{\prime}-t^{\prime \prime}\right)=2 \int_{0}^{t} d s(t-s) f(s),
$$

we find, for $t \rightarrow+\infty$, that

$$
D_{i j}=\int_{0}^{+\infty}\left\langle\frac{\partial \delta \Phi}{\partial w_{i}}(\mathbf{w}, \mathbf{J}, 0) \frac{\partial \delta \Phi}{\partial w_{j}}(\mathbf{w}+\boldsymbol{\Omega} s, \mathbf{J}, s)\right\rangle d s .
$$

This is the Kubo formula for our problem. Replacing the correlation function by its expression (74), we get

$$
D_{i j}=(2 \pi)^{d} m \int_{0}^{+\infty} d s \sum_{\mathbf{k}, \mathbf{k}^{\prime}} \int d \mathbf{J}^{\prime} k_{i} k_{j} e^{i\left(\mathbf{k} \cdot \boldsymbol{\Omega}-\mathbf{k}^{\prime} \cdot \mathbf{\Omega}^{\prime}\right) s} \frac{1}{\left|D_{\mathbf{k}, \mathbf{k}^{\prime}}\left(\mathbf{J}, \mathbf{J}^{\prime}, \mathbf{k}^{\prime} \cdot \mathbf{\Omega}^{\prime}\right)\right|^{2}} f\left(\mathbf{J}^{\prime}\right)
$$

Making the change of variables $s \rightarrow-s$ and $\left(\mathbf{k}, \mathbf{k}^{\prime}\right) \rightarrow\left(-\mathbf{k},-\mathbf{k}^{\prime}\right)$, we see that we can replace $\int_{0}^{+\infty} d s$ by $(1 / 2) \int_{-\infty}^{+\infty} d s$. Then, using the identity

$$
\delta(\omega)=\int_{-\infty}^{+\infty} e^{i \omega t} \frac{d t}{2 \pi}
$$

we obtain the final expression

$$
D_{i j}=\pi(2 \pi)^{d} m \sum_{\mathbf{k}, \mathbf{k}^{\prime}} \int d \mathbf{J}^{\prime} k_{i} k_{j} \frac{1}{\left|D_{\mathbf{k}, \mathbf{k}^{\prime}}\left(\mathbf{J}, \mathbf{J}^{\prime}, \mathbf{k}^{\prime} \cdot \mathbf{\Omega}^{\prime}\right)\right|^{2}} \delta\left(\mathbf{k} \cdot \boldsymbol{\Omega}-\mathbf{k}^{\prime} \cdot \mathbf{\Omega}^{\prime}\right) f\left(\mathbf{J}^{\prime}\right) .
$$

\section{The friction due to the polarization}

We now compute the friction term defined by Eq. (69). We need to keep terms up to order 1/N. From Eq. (66), the first term to compute is

$$
\mathbf{A}_{I}=-\frac{1}{t} \int_{0}^{t} d t^{\prime}\left\langle\frac{\partial \delta \Phi}{\partial \mathbf{w}}\left(\mathbf{w}+\boldsymbol{\Omega} t^{\prime}, \mathbf{J}, t^{\prime}\right)\right\rangle .
$$

By the inverse Fourier-Laplace transform, we have

$$
\left\langle\frac{\partial \delta \Phi}{\partial \mathbf{w}}\left(\mathbf{w}+\boldsymbol{\Omega} t^{\prime}, \mathbf{J}, t^{\prime}\right)\right\rangle=i \sum_{\mathbf{k}} \int_{\mathcal{C}} \frac{d \omega}{2 \pi} \mathbf{k} e^{i \mathbf{k} \cdot\left(\mathbf{w}+\boldsymbol{\Omega} t^{\prime}\right)} e^{-i \omega t^{\prime}}\langle\delta \tilde{\Phi}(\mathbf{k}, \mathbf{J}, \omega)\rangle .
$$

Using Eq. (37), we find that

$$
\langle\delta \tilde{\Phi}(\mathbf{k}, \mathbf{J}, \omega)\rangle=-(2 \pi)^{d} \sum_{\mathbf{k}^{\prime}} \int d \mathbf{J}^{\prime} \frac{1}{D_{\mathbf{k}, \mathbf{k}^{\prime}}\left(\mathbf{J}, \mathbf{J}^{\prime}, \omega\right)} \frac{\left\langle\delta \hat{f}\left(\mathbf{k}^{\prime}, \mathbf{J}^{\prime}, 0\right)\right\rangle}{i\left(\mathbf{k}^{\prime} \cdot \mathbf{\Omega}^{\prime}-\omega\right)}
$$


Now, using the fact that the test particle is initially located in $(\mathbf{w}, \mathbf{J})$, so that $\left\langle\delta f\left(\mathbf{w}^{\prime}, \mathbf{J}^{\prime}, 0\right)\right\rangle=m \delta\left(\mathbf{w}^{\prime}-\mathbf{w}\right) \delta\left(\mathbf{J}^{\prime}-\mathbf{J}\right)$, we obtain from Eq. (20) the result

$$
\left\langle\delta \hat{f}\left(\mathbf{k}^{\prime}, \mathbf{J}^{\prime}, 0\right)\right\rangle=\frac{1}{(2 \pi)^{d}} m e^{-i \mathbf{k}^{\prime} \cdot \mathbf{w}} \delta\left(\mathbf{J}^{\prime}-\mathbf{J}\right) .
$$

Substituting these expressions in Eq. (81), and averaging over $\mathbf{w}$ (which amounts to replacing $\mathbf{k}^{\prime}$ by $\mathbf{k}$ ), we get

$$
\left\langle\frac{\partial \delta \Phi}{\partial \mathbf{w}}\left(\mathbf{w}+\boldsymbol{\Omega} t^{\prime}, \mathbf{J}, t^{\prime}\right)\right\rangle=-m \sum_{\mathbf{k}} \int_{\mathcal{C}} \frac{d \omega}{2 \pi} \mathbf{k} e^{i(\mathbf{k} \cdot \boldsymbol{\Omega}-\omega) t^{\prime}} \frac{1}{D_{\mathbf{k}, \mathbf{k}}(\mathbf{J}, \mathbf{J}, \omega)} \frac{1}{\mathbf{k} \cdot \boldsymbol{\Omega}-\omega} .
$$

Therefore, the friction term (80) is given by

$$
\mathbf{A}_{I}=m \frac{1}{t} \int_{0}^{t} d t^{\prime} \sum_{\mathbf{k}} \int_{\mathcal{C}} \frac{d \omega}{2 \pi} \mathbf{k} e^{i(\mathbf{k} \cdot \boldsymbol{\Omega}-\omega) t^{\prime}} \frac{1}{D_{\mathbf{k}, \mathbf{k}}(\mathbf{J}, \mathbf{J}, \omega)} \frac{1}{\mathbf{k} \cdot \boldsymbol{\Omega}-\omega}
$$

We now use the Landau prescription $\omega \rightarrow \omega+i 0^{+}$and the Plemelj formula (46) to evaluate the integral over $\omega$. The term corresponding to the imaginary part in the Plemelj formula is

$$
\mathbf{A}_{I}^{(a)}=i m \pi \frac{1}{t} \int_{0}^{t} d t^{\prime} \sum_{\mathbf{k}} \int_{\mathcal{C}} \frac{d \omega}{2 \pi} \mathbf{k} e^{i(\mathbf{k} \cdot \boldsymbol{\Omega}-\omega) t^{\prime}} \frac{1}{D_{\mathbf{k}, \mathbf{k}}(\mathbf{J}, \mathbf{J}, \omega)} \delta(\mathbf{k} \cdot \boldsymbol{\Omega}-\omega) .
$$

Integrating over $\omega$ and $t^{\prime}$, we obtain

$$
\mathbf{A}_{I}^{(a)}=-\frac{m}{2} \sum_{\mathbf{k}} \mathbf{k} \operatorname{Im}\left(\frac{1}{D_{\mathbf{k}, \mathbf{k}}(\mathbf{J}, \mathbf{J}, \mathbf{k} \cdot \boldsymbol{\Omega})}\right) .
$$

The term corresponding to the real part in the Plemelj formula is

$$
\mathbf{A}_{I}^{(b)}=m \frac{1}{t} \int_{0}^{t} d t^{\prime} \sum_{\mathbf{k}} \mathcal{P} \int_{-\infty}^{+\infty} \frac{d \omega}{2 \pi} \mathbf{k} e^{i(\mathbf{k} \cdot \boldsymbol{\Omega}-\omega) t^{\prime}} \frac{1}{D_{\mathbf{k}, \mathbf{k}}(\mathbf{J}, \mathbf{J}, \omega)} \frac{1}{\mathbf{k} \cdot \boldsymbol{\Omega}-\omega} .
$$

Integrating over $t^{\prime}$, we can convert this expression to the form

$$
\mathbf{A}_{I}^{(b)}=-i m \sum_{\mathbf{k}} \mathcal{P} \int_{-\infty}^{+\infty} \frac{d \omega}{2 \pi} \mathbf{k} \frac{1}{D_{\mathbf{k}, \mathbf{k}}(\mathbf{J}, \mathbf{J}, \omega)} \frac{1}{(\mathbf{k} \cdot \boldsymbol{\Omega}-\omega)^{2}} \frac{1}{t}\{i \sin [(\mathbf{k} \cdot \boldsymbol{\Omega}-\omega) t]+\cos [(\mathbf{k} \cdot \boldsymbol{\Omega}-\omega) t]-1\} .
$$

For $t \rightarrow+\infty$, using the identity

$$
\lim _{t \rightarrow+\infty} \frac{1-\cos (t x)}{t x^{2}}=\pi \delta(x),
$$

and integrating over $\omega$, we find that $\mathbf{A}_{I}^{(b)}$ is given by Eq. (87), just like $\mathbf{A}_{I}^{(a)}$. Therefore, writing $\mathbf{A}_{I}=\mathbf{A}_{I}^{(a)}+\mathbf{A}_{I}^{(b)}=$ $2 \mathbf{A}_{I}^{(a)}$, we obtain

$$
\mathbf{A}_{I}=-m \sum_{\mathbf{k}} \mathbf{k} \operatorname{Im}\left(\frac{1}{D_{\mathbf{k}, \mathbf{k}}(\mathbf{J}, \mathbf{J}, \mathbf{k} \cdot \boldsymbol{\Omega})}\right)
$$

Finally, using Eq. (54), we find that

$$
\mathbf{A}_{I}=\pi(2 \pi)^{d} m \sum_{\mathbf{k}, \mathbf{k}^{\prime}} \int d \mathbf{J}^{\prime} \mathbf{k}\left(\mathbf{k}^{\prime} \cdot \frac{\partial f^{\prime}}{\partial \mathbf{J}^{\prime}}\right) \delta\left(\mathbf{k} \cdot \boldsymbol{\Omega}-\mathbf{k}^{\prime} \cdot \boldsymbol{\Omega}^{\prime}\right) \frac{1}{\left|D_{\mathbf{k}, \mathbf{k}^{\prime}}\left(\mathbf{J}, \mathbf{J}^{\prime}, \mathbf{k} \cdot \boldsymbol{\Omega}\right)\right|^{2}} .
$$

As we shall see, $\mathbf{A}_{I}$ corresponds to the friction due to the polarization denoted $\mathbf{F}_{p o l}$ in Eq. (71).

Remark: We can obtain Eq. (92) in a slightly more direct manner from Eq. (84) by using the contour of integration shown in Fig. 9 of [72]. In that case, the integral over $\omega$ is just $-2 \pi i$ times the sum of the residues at the poles of the integrand in Eq. (84). The poles corresponding to the zeros of $D_{\mathbf{k}, \mathbf{k}}(\mathbf{J}, \mathbf{J}, \omega)$ give a contribution that rapidly decays with time since $\operatorname{Im}(\omega)<0$ (the system is Vlasov stable). Keeping only the contribution of the pole $\omega=\mathbf{k} \cdot \boldsymbol{\Omega}$ that does not decay in time we obtain

$$
\left\langle\frac{\partial \delta \Phi}{\partial \mathbf{w}}\left(\mathbf{w}+\boldsymbol{\Omega} t^{\prime}, \mathbf{J}, t^{\prime}\right)\right\rangle=-i m \sum_{\mathbf{k}} \mathbf{k} \frac{1}{D_{\mathbf{k}, \mathbf{k}}(\mathbf{J}, \mathbf{J}, \mathbf{k} \cdot \boldsymbol{\Omega})} .
$$

Substituting this result in Eq. (80), we get Eq. (91) then Eq. (92). 


\section{The part of the friction due to the inhomogeneity of the diffusion coefficient}

In the evaluation of the total friction, at the order $1 / N$, the second term to compute is

$$
A_{i}^{I I}=\frac{1}{t} \int_{0}^{t} d t^{\prime} \int_{0}^{t^{\prime}} d t^{\prime \prime} \int_{0}^{t^{\prime \prime}} d t^{\prime \prime \prime} \frac{\partial \Omega_{l}}{\partial J_{j}}\left\langle\frac{\partial^{2} \delta \Phi}{\partial w_{i} \partial w_{l}}\left(\mathbf{w}+\boldsymbol{\Omega} t^{\prime}, \mathbf{J}, t^{\prime}\right) \frac{\partial \delta \Phi}{\partial w_{j}}\left(\mathbf{w}+\boldsymbol{\Omega} t^{\prime \prime \prime}, \mathbf{J}, t^{\prime \prime \prime}\right)\right\rangle .
$$

By the inverse Fourier-Laplace transform, we have

$$
\begin{aligned}
\left\langle\frac{\partial^{2} \delta \Phi}{\partial w_{i} \partial w_{l}}\left(\mathbf{w}+\boldsymbol{\Omega} t^{\prime}, \mathbf{J}, t^{\prime}\right) \frac{\partial \delta \Phi}{\partial w_{j}}\left(\mathbf{w}+\boldsymbol{\Omega} t^{\prime \prime \prime}, \mathbf{J}, t^{\prime \prime \prime}\right)\right\rangle= & -i \sum_{\mathbf{k}} \int_{\mathcal{C}} \frac{d \omega}{2 \pi} \sum_{\mathbf{k}^{\prime}} \int_{\mathcal{C}} \frac{d \omega^{\prime}}{2 \pi} k_{i} k_{l} k_{j}^{\prime} e^{i \mathbf{k} \cdot\left(\mathbf{w}+\boldsymbol{\Omega} t^{\prime}\right)} e^{-i \omega t^{\prime}} \\
& \times e^{i \mathbf{k}^{\prime}\left(\mathbf{w}+\boldsymbol{\Omega} t^{\prime \prime \prime}\right)} e^{-i \omega^{\prime} t^{\prime \prime \prime}}\left\langle\delta \tilde{\Phi}(\mathbf{k}, \mathbf{J}, \omega) \delta \tilde{\Phi}\left(\mathbf{k}^{\prime}, \mathbf{J}, \omega^{\prime}\right)\right\rangle
\end{aligned}
$$

Substituting Eq. (43) in Eq. (95), averaging over w (which amounts to replacing $\mathbf{k}^{\prime}$ by $-\mathbf{k}$ ), and carrying out the integrals over $\omega^{\prime}$ and $\omega$, we end up with the result

$$
\begin{array}{r}
\left\langle\frac{\partial^{2} \delta \Phi}{\partial w_{i} \partial w_{l}}\left(\mathbf{w}+\boldsymbol{\Omega} t^{\prime}, \mathbf{J}, t^{\prime}\right) \frac{\partial \delta \Phi}{\partial w_{j}}\left(\mathbf{w}+\boldsymbol{\Omega} t^{\prime \prime \prime}, \mathbf{J}, t^{\prime \prime \prime}\right)\right\rangle=i(2 \pi)^{d} m \sum_{\mathbf{k}, \mathbf{k}^{\prime}} \int d \mathbf{J}^{\prime} k_{i} k_{j} k_{l} e^{i\left(\mathbf{k} \cdot \boldsymbol{\Omega}-\mathbf{k}^{\prime} \cdot \mathbf{\Omega}^{\prime}\right)\left(t^{\prime}-t^{\prime \prime \prime}\right)} \\
\times \frac{1}{\left|D_{\mathbf{k}, \mathbf{k}^{\prime}}\left(\mathbf{J}, \mathbf{J}^{\prime}, \mathbf{k}^{\prime} \cdot \mathbf{\Omega}^{\prime}\right)\right|^{2}} f\left(\mathbf{J}^{\prime}\right)
\end{array}
$$

This expression shows that the correlation function appearing in Eq. (94) is an odd function of $t^{\prime}-t^{\prime \prime \prime}$. Using the identity

$$
\int_{0}^{t^{\prime}} d t^{\prime \prime} \int_{0}^{t^{\prime \prime}} d t^{\prime \prime \prime} f\left(t^{\prime}-t^{\prime \prime \prime}\right)=\int_{0}^{t^{\prime}} d t^{\prime \prime \prime}\left(t^{\prime}-t^{\prime \prime \prime}\right) f\left(t^{\prime}-t^{\prime \prime \prime}\right)
$$

we find that

$$
A_{i}^{I I}=i(2 \pi)^{d} m \frac{1}{t} \int_{0}^{t} d t^{\prime} \int_{0}^{t^{\prime}} d t^{\prime \prime \prime} \sum_{\mathbf{k}, \mathbf{k}^{\prime}} \int d \mathbf{J}^{\prime} k_{i} k_{j} k_{l} \frac{\partial \Omega_{l}}{\partial J_{j}}\left(t^{\prime}-t^{\prime \prime \prime}\right) e^{i\left(\mathbf{k} \cdot \boldsymbol{\Omega}-\mathbf{k}^{\prime} \cdot \mathbf{\Omega}^{\prime}\right)\left(t^{\prime}-t^{\prime \prime \prime}\right)} \frac{1}{\left|D_{\mathbf{k}, \mathbf{k}^{\prime}}\left(\mathbf{J}, \mathbf{J}^{\prime}, \mathbf{k}^{\prime} \cdot \boldsymbol{\Omega}^{\prime}\right)\right|^{2}} f\left(\mathbf{J}^{\prime}\right) .
$$

This can be rewritten

$$
A_{i}^{I I}=(2 \pi)^{d} m \frac{1}{t} \int_{0}^{t} d t^{\prime} \int_{0}^{t^{\prime}} d t^{\prime \prime \prime} \sum_{\mathbf{k}, \mathbf{k}^{\prime}} \int d \mathbf{J}^{\prime} k_{i} k_{j} \frac{\partial}{\partial J_{j}}\left(e^{i\left(\mathbf{k} \cdot \boldsymbol{\Omega}-\mathbf{k}^{\prime} \cdot \boldsymbol{\Omega}^{\prime}\right)\left(t^{\prime}-t^{\prime \prime \prime}\right)}\right) \frac{1}{\left|D_{\mathbf{k}, \mathbf{k}^{\prime}}\left(\mathbf{J}, \mathbf{J}^{\prime}, \mathbf{k}^{\prime} \cdot \mathbf{\Omega}^{\prime}\right)\right|^{2}} f\left(\mathbf{J}^{\prime}\right),
$$

or, equivalently,

$$
\begin{aligned}
& A_{i}^{I I}=(2 \pi)^{d} m \frac{1}{t} \frac{\partial}{\partial J_{j}} \int_{0}^{t} d t^{\prime} \int_{0}^{t^{\prime}} d t^{\prime \prime \prime} \sum_{\mathbf{k}, \mathbf{k}^{\prime}} \int d \mathbf{J}^{\prime} k_{i} k_{j} e^{i\left(\mathbf{k} \cdot \boldsymbol{\Omega}-\mathbf{k}^{\prime} \cdot \boldsymbol{\Omega}^{\prime}\right)\left(t^{\prime}-t^{\prime \prime \prime}\right)} \frac{1}{\left|D_{\mathbf{k}, \mathbf{k}_{1}}\left(\mathbf{J}, \mathbf{J}^{\prime}, \mathbf{k}^{\prime} \cdot \mathbf{\Omega}^{\prime}\right)\right|^{2}} f\left(\mathbf{J}^{\prime}\right) \\
& -(2 \pi)^{d} m \frac{1}{t} \int_{0}^{t} d t^{\prime} \int_{0}^{t^{\prime}} d t^{\prime \prime \prime} \sum_{\mathbf{k}, \mathbf{k}^{\prime}} \int d \mathbf{J}^{\prime} k_{i} k_{j} e^{i\left(\mathbf{k} \cdot \boldsymbol{\Omega}-\mathbf{k}^{\prime} \cdot \boldsymbol{\Omega}^{\prime}\right)\left(t^{\prime}-t^{\prime \prime \prime}\right)} \frac{\partial}{\partial J_{j}}\left(\frac{1}{\left|D_{\mathbf{k}, \mathbf{k}^{\prime}}\left(\mathbf{J}, \mathbf{J}^{\prime}, \mathbf{k}^{\prime} \cdot \boldsymbol{\Omega}^{\prime}\right)\right|^{2}}\right) f\left(\mathbf{J}^{\prime}\right)
\end{aligned}
$$

Since the integrand only depends on $t^{\prime}-t^{\prime \prime \prime}$, using the identity (75), we obtain for $t \rightarrow+\infty$,

$$
\begin{aligned}
& A_{i}^{I I}=(2 \pi)^{d} m \frac{\partial}{\partial J_{j}} \int_{0}^{+\infty} d s \sum_{\mathbf{k}, \mathbf{k}^{\prime}} \int d \mathbf{J}^{\prime} k_{i} k_{j} e^{i\left(\mathbf{k} \cdot \boldsymbol{\Omega}-\mathbf{k}^{\prime} \cdot \mathbf{\Omega}^{\prime}\right) s} \frac{1}{\left|D_{\mathbf{k}, \mathbf{k}^{\prime}}\left(\mathbf{J}, \mathbf{J}^{\prime}, \mathbf{k}^{\prime} \cdot \mathbf{\Omega}^{\prime}\right)\right|^{2}} f\left(\mathbf{J}^{\prime}\right) \\
& -(2 \pi)^{d} m \int_{0}^{+\infty} d s \sum_{\mathbf{k}, \mathbf{k}^{\prime}} \int d \mathbf{J}^{\prime} k_{i} k_{j} e^{i\left(\mathbf{k} \cdot \boldsymbol{\Omega}-\mathbf{k}^{\prime} \cdot \boldsymbol{\Omega}^{\prime}\right) s} \frac{\partial}{\partial J_{j}}\left(\frac{1}{\left|D_{\mathbf{k}, \mathbf{k}^{\prime}}\left(\mathbf{J}, \mathbf{J}^{\prime}, \mathbf{k}^{\prime} \cdot \mathbf{\Omega}^{\prime}\right)\right|^{2}}\right) f\left(\mathbf{J}^{\prime}\right) .
\end{aligned}
$$

Making the change of variables $s \rightarrow-s$ and $\left(\mathbf{k}, \mathbf{k}^{\prime}\right) \rightarrow\left(-\mathbf{k},-\mathbf{k}^{\prime}\right)$, we see that we can replace $\int_{0}^{+\infty} d s$ by $(1 / 2) \int_{-\infty}^{+\infty} d s$. Then, using the identity (78), we obtain the expression

$$
\begin{aligned}
& A_{i}^{I I}=\pi(2 \pi)^{d} m \frac{\partial}{\partial J_{j}} \sum_{\mathbf{k}, \mathbf{k}^{\prime}} \int d \mathbf{J}^{\prime} k_{i} k_{j} \frac{1}{\left|D_{\mathbf{k}, \mathbf{k}^{\prime}}\left(\mathbf{J}, \mathbf{J}^{\prime}, \mathbf{k}^{\prime} \cdot \boldsymbol{\Omega}^{\prime}\right)\right|^{2}} \delta\left(\mathbf{k} \cdot \boldsymbol{\Omega}-\mathbf{k}^{\prime} \cdot \mathbf{\Omega}^{\prime}\right) f\left(\mathbf{J}^{\prime}\right) \\
& -\pi(2 \pi)^{d} m \sum_{\mathbf{k}, \mathbf{k}^{\prime}} \int d \mathbf{J}^{\prime} k_{i} k_{j} \frac{\partial}{\partial J_{j}}\left(\frac{1}{\left|D_{\mathbf{k}, \mathbf{k}^{\prime}}\left(\mathbf{J}, \mathbf{J}^{\prime}, \mathbf{k}^{\prime} \cdot \mathbf{\Omega}^{\prime}\right)\right|^{2}}\right) \delta\left(\mathbf{k} \cdot \boldsymbol{\Omega}-\mathbf{k}^{\prime} \cdot \mathbf{\Omega}^{\prime}\right) f\left(\mathbf{J}^{\prime}\right) .
\end{aligned}
$$


In the first term, we recover the diffusion coefficient (79) so that

$$
A_{i}^{I I}=\frac{\partial D_{i j}}{\partial J_{j}}-\pi(2 \pi)^{d} m \sum_{\mathbf{k}, \mathbf{k}^{\prime}} \int d \mathbf{J}^{\prime} k_{i} k_{j} \delta\left(\mathbf{k} \cdot \boldsymbol{\Omega}-\mathbf{k}^{\prime} \cdot \boldsymbol{\Omega}^{\prime}\right) \frac{\partial}{\partial J_{j}}\left(\frac{1}{\left|D_{\mathbf{k}, \mathbf{k}^{\prime}}\left(\mathbf{J}, \mathbf{J}^{\prime}, \mathbf{k}^{\prime} \cdot \mathbf{\Omega}^{\prime}\right)\right|^{2}}\right) f\left(\mathbf{J}^{\prime}\right) .
$$

Finally, the third and fourth terms to compute are

$$
A_{i}^{I I I}=\frac{1}{t} \int_{0}^{t} d t^{\prime} \int_{0}^{t^{\prime}} d t^{\prime \prime}\left\langle\frac{\partial^{2} \delta \Phi}{\partial w_{i} \partial J_{j}}\left(\mathbf{w}+\boldsymbol{\Omega} t^{\prime}, \mathbf{J}, t^{\prime}\right) \frac{\partial \delta \Phi}{\partial w_{j}}\left(\mathbf{w}+\boldsymbol{\Omega} t^{\prime \prime}, \mathbf{J}, t^{\prime \prime}\right)\right\rangle,
$$

and

$$
A_{i}^{I V}=-\frac{1}{t} \int_{0}^{t} d t^{\prime} \int_{0}^{t^{\prime}} d t^{\prime \prime}\left\langle\frac{\partial^{2} \delta \Phi}{\partial w_{i} \partial w_{j}}\left(\mathbf{w}+\boldsymbol{\Omega} t^{\prime}, \mathbf{J}, t^{\prime}\right) \frac{\partial \delta \Phi}{\partial J_{j}}\left(\mathbf{w}+\boldsymbol{\Omega} t^{\prime \prime}, \mathbf{J}, t^{\prime \prime}\right)\right\rangle .
$$

Substituting the inverse Fourier-Laplace transform of the fluctuations of the potential in these equations, averaging over $\mathbf{w}$ (which amounts to replacing $\mathbf{k}^{\prime}$ by $-\mathbf{k}$ ) and summing the resulting expressions, we obtain

$$
A_{i}^{I I I}+A_{i}^{I V}=\frac{1}{t} \int_{0}^{t} d t^{\prime} \int_{0}^{t^{\prime}} d t^{\prime \prime} \sum_{\mathbf{k}} \int_{\mathcal{C}} \frac{d \omega}{2 \pi} \int_{\mathcal{C}} \frac{d \omega^{\prime}}{2 \pi} k_{i} k_{j} e^{i \mathbf{k} \cdot \boldsymbol{\Omega} t^{\prime}} e^{-i \omega t^{\prime}} e^{-i \mathbf{k} \cdot \boldsymbol{\Omega} t^{\prime \prime}} e^{-i \omega^{\prime} t^{\prime \prime}} \frac{\partial}{\partial J_{j}}\left\langle\delta \tilde{\Phi}(\mathbf{k}, \mathbf{J}, \omega) \delta \tilde{\Phi}\left(-\mathbf{k}, \mathbf{J}, \omega^{\prime}\right)\right\rangle .
$$

Substituting Eq. (43) in Eq. (106), and carrying out the integrals over $\omega^{\prime}$ and $\omega$, we end up with the result

$$
A_{i}^{I I I}+A_{i}^{I V}=(2 \pi)^{d} m \frac{1}{t} \int_{0}^{t} d t^{\prime} \int_{0}^{t^{\prime}} d t^{\prime \prime} \sum_{\mathbf{k}, \mathbf{k}^{\prime}} \int d \mathbf{J}^{\prime} k_{i} k_{j} e^{i\left(\mathbf{k} \cdot \boldsymbol{\Omega}-\mathbf{k}^{\prime} \cdot \boldsymbol{\Omega}^{\prime}\right)\left(t^{\prime}-t^{\prime \prime}\right)} \frac{\partial}{\partial J_{j}}\left(\frac{1}{\left|D_{\mathbf{k}, \mathbf{k}^{\prime}}\left(\mathbf{J}, \mathbf{J}^{\prime}, \mathbf{k}^{\prime} \cdot \Omega^{\prime}\right)\right|^{2}}\right) f\left(\mathbf{J}^{\prime}\right) .
$$

This expression shows that the correlation function appearing under the integral sign only depends on $t^{\prime}-t^{\prime \prime}$. Using the identity (75) we find, for $t \rightarrow+\infty$, that

$$
A_{i}^{I I I}+A_{i}^{I V}=(2 \pi)^{d} m \int_{0}^{+\infty} d s \sum_{\mathbf{k}, \mathbf{k}^{\prime}} \int d \mathbf{J}^{\prime} k_{i} k_{j} e^{i\left(\mathbf{k} \cdot \boldsymbol{\Omega}-\mathbf{k}^{\prime} \cdot \mathbf{\Omega}^{\prime}\right) s} \frac{\partial}{\partial J_{j}}\left(\frac{1}{\left|D_{\mathbf{k}, \mathbf{k}^{\prime}}\left(\mathbf{J}, \mathbf{J}^{\prime}, \mathbf{k}^{\prime} \cdot \Omega^{\prime}\right)\right|^{2}}\right) f\left(\mathbf{J}^{\prime}\right) .
$$

Making the change of variables $s \rightarrow-s$ and $\left(\mathbf{k}, \mathbf{k}^{\prime}\right) \rightarrow\left(-\mathbf{k},-\mathbf{k}^{\prime}\right)$, we see that we can replace $\int_{0}^{+\infty} d s$ by $(1 / 2) \int_{-\infty}^{+\infty} d s$. Then, using the identity (78), we obtain the expression

$$
A_{i}^{I I I}+A_{i}^{I V}=\pi(2 \pi)^{d} m \sum_{\mathbf{k}, \mathbf{k}^{\prime}} \int d \mathbf{J}^{\prime} k_{i} k_{j} \frac{\partial}{\partial J_{j}}\left(\frac{1}{\left|D_{\mathbf{k}, \mathbf{k}^{\prime}}\left(\mathbf{J}, \mathbf{J}^{\prime}, \mathbf{k}^{\prime} \cdot \Omega^{\prime}\right)\right|^{2}}\right) \delta\left(\mathbf{k} \cdot \mathbf{\Omega}-\mathbf{k}^{\prime} \cdot \mathbf{\Omega}^{\prime}\right) f\left(\mathbf{J}^{\prime}\right) .
$$

Finally, summing Eqs. (103) and (109), we get

$$
A_{I I}+A_{I I I}+A_{I V}=\frac{\partial D_{i j}}{\partial J_{j}}
$$

\section{E. Connection between the kinetic equation (56) and the Fokker-Planck equation (70)}

We have established that the diffusion tensor and the friction force are given by

$$
\begin{gathered}
D_{i j}=\pi(2 \pi)^{d} m \sum_{\mathbf{k}, \mathbf{k}^{\prime}} \int d \mathbf{J}^{\prime} k_{i} k_{j} \frac{1}{\left|D_{\mathbf{k}, \mathbf{k}^{\prime}}\left(\mathbf{J}, \mathbf{J}^{\prime}, \mathbf{k}^{\prime} \cdot \mathbf{\Omega}^{\prime}\right)\right|^{2}} \delta\left(\mathbf{k} \cdot \boldsymbol{\Omega}-\mathbf{k}^{\prime} \cdot \mathbf{\Omega}^{\prime}\right) f\left(\mathbf{J}^{\prime}\right) . \\
A_{i}=\pi(2 \pi)^{d} m \sum_{\mathbf{k}, \mathbf{k}^{\prime}} \int d \mathbf{J}^{\prime} k_{i} \frac{1}{\left|D_{\mathbf{k}, \mathbf{k}^{\prime}}\left(\mathbf{J}, \mathbf{J}^{\prime}, \mathbf{k} \cdot \boldsymbol{\Omega}\right)\right|^{2}} \delta\left(\mathbf{k} \cdot \boldsymbol{\Omega}-\mathbf{k}^{\prime} \cdot \mathbf{\Omega}^{\prime}\right)\left(\mathbf{k}^{\prime} \cdot \frac{\partial f^{\prime}}{\partial \mathbf{J}^{\prime}}\right)+\frac{\partial D_{i j}}{\partial J_{j}} .
\end{gathered}
$$

Comparing Eq. (112) with Eq. (71), we see that the friction by polarization is

$$
\mathbf{F}_{p o l}=\pi(2 \pi)^{d} m \sum_{\mathbf{k}, \mathbf{k}^{\prime}} \int d \mathbf{J}^{\prime} \mathbf{k} \frac{1}{\left|D_{\mathbf{k}, \mathbf{k}^{\prime}}\left(\mathbf{J}, \mathbf{J}^{\prime}, \mathbf{k} \cdot \boldsymbol{\Omega}\right)\right|^{2}} \delta\left(\mathbf{k} \cdot \boldsymbol{\Omega}-\mathbf{k}^{\prime} \cdot \boldsymbol{\Omega}^{\prime}\right)\left(\mathbf{k}^{\prime} \cdot \frac{\partial f^{\prime}}{\partial \mathbf{J}^{\prime}}\right) .
$$


On the other hand, using an integration by part in the first term of Eq. (112), the total friction can be written

$$
\mathbf{A}=\pi(2 \pi)^{d} m \sum_{\mathbf{k}, \mathbf{k}^{\prime}} \int d \mathbf{J}^{\prime} f\left(\mathbf{J}^{\prime}\right)\left(\mathbf{k} \cdot \frac{\partial}{\partial \mathbf{J}}-\mathbf{k}^{\prime} \cdot \frac{\partial}{\partial \mathbf{J}^{\prime}}\right) \frac{1}{\left|D_{\mathbf{k}, \mathbf{k}^{\prime}}\left(\mathbf{J}, \mathbf{J}^{\prime}, \mathbf{k} \cdot \boldsymbol{\Omega}\right)\right|^{2}} \delta\left(\mathbf{k} \cdot \boldsymbol{\Omega}-\mathbf{k}^{\prime} \cdot \boldsymbol{\Omega}^{\prime}\right)
$$

Finally, using Eqs. (111) and (113), we find that the Fokker-Planck equation (70) becomes

$$
\frac{\partial P}{\partial t}=\pi(2 \pi)^{d} m \frac{\partial}{\partial \mathbf{J}} \cdot \sum_{\mathbf{k}, \mathbf{k}^{\prime}} \int d \mathbf{J}^{\prime} \mathbf{k} \frac{1}{\left|D_{\mathbf{k}, \mathbf{k}^{\prime}}\left(\mathbf{J}, \mathbf{J}^{\prime}, \mathbf{k} \cdot \boldsymbol{\Omega}\right)\right|^{2}} \delta\left(\mathbf{k} \cdot \boldsymbol{\Omega}-\mathbf{k}^{\prime} \cdot \boldsymbol{\Omega}^{\prime}\right)\left(\mathbf{k} \cdot \frac{\partial}{\partial \mathbf{J}}-\mathbf{k}^{\prime} \cdot \frac{\partial}{\partial \mathbf{J}^{\prime}}\right) P(\mathbf{J}, t) f\left(\mathbf{J}^{\prime}\right)
$$

When collective effects are neglected, we recover the results obtained in 67, 68] by a different method (related expressions of the diffusion and friction terms have also been obtained in [59 66]). For spatially homogeneous systems, we recover the results of Hubbard [11] (see also [37] when collective effects are neglected). As observed in our previous works, we note that the form of Eq. (115) is very similar to the form of Eq. (56). This shows that the FokkerPlanck equation (115), with the diffusion coefficient (111) and the friction (113), can be directly obtained from the Lenard-Balescu-type equation (56) by replacing the time dependent distribution $f\left(\mathbf{J}^{\prime}, t\right)$ by the static distribution $f\left(\mathbf{J}^{\prime}\right)$ of the bath. This procedure transforms an integro-differential equation (56) into a differential equation (115). Although natural, the rigorous justification of this procedure requires the detailed calculation that we have given here. In fact, we can understand this result in the following manner. Equations (56) and (115) govern the evolution of the distribution function of a test particle (described by the coordinate $\mathbf{J}$ ) interacting with field particles (described by the running coordinate $\mathbf{J}^{\prime}$ ). In Eq. (56), all the particles are equivalent so the distribution of the field particles $f\left(\mathbf{J}^{\prime}, t\right)$ changes with time exactly like the distribution of the test particle $f(\mathbf{J}, t)$. In Eq. (115), the test particle and the field particles are not equivalent since the field particles form a "bath". The field particles have a steady (given) distribution $f\left(\mathbf{J}^{\prime}\right)$ while the distribution of the test particle $f(\mathbf{J}, t)=N m P(\mathbf{J}, t)$ changes with time.

\section{F. Thermal bath: Boltzmann distribution}

For a thermal bath, the field particles have the Boltzmann distribution of statistical equilibrium

$$
f(\mathbf{J})=A e^{-\beta m H(\mathbf{J})},
$$

where $H(\mathbf{J})$ is the individual energy of the particles. We note the identity

$$
\frac{\partial f}{\partial \mathbf{J}}=-\beta m f(\mathbf{J}) \boldsymbol{\Omega}(\mathbf{J})
$$

where we have used $\partial H / \partial \mathbf{J}=\boldsymbol{\Omega}(\mathbf{J})$. Substituting this relation in Eq. (113), we obtain

$$
\mathbf{F}_{p o l}=-\pi(2 \pi)^{d} m^{2} \beta \sum_{\mathbf{k}, \mathbf{k}^{\prime}} \int d \mathbf{J}^{\prime} \mathbf{k} \frac{1}{\left|D_{\mathbf{k}, \mathbf{k}^{\prime}}\left(\mathbf{J}, \mathbf{J}^{\prime}, \mathbf{k} \cdot \boldsymbol{\Omega}\right)\right|^{2}} \delta\left(\mathbf{k} \cdot \boldsymbol{\Omega}-\mathbf{k}^{\prime} \cdot \boldsymbol{\Omega}^{\prime}\right)\left(\mathbf{k}^{\prime} \cdot \mathbf{\Omega}^{\prime}\right) f\left(\mathbf{J}^{\prime}\right) .
$$

Using the $\delta$-function to replace $\mathbf{k}^{\prime} \cdot \Omega^{\prime}$ by $\mathbf{k} \cdot \Omega$, and comparing the resulting expression with Eq. (111), we find that

$$
F_{i}^{p o l}=-\beta m D_{i j}(\mathbf{J}) \Omega_{j}(\mathbf{J})
$$

This can be viewed as a generalized Einstein relation connecting the friction force to the diffusion tensor (fluctuationdissipation theorem). We stress that the Einstein relation is valid for the friction by polarization $\mathbf{F}_{p o l}$, not for the total friction $\mathbf{A}$ that has a more complex expression due to the term $\partial_{j} D_{i j}$. We do not have this subtlety for the usual Brownian motion where the diffusion coefficient is constant. For a thermal bath, using Eq. (119), the Fokker-Planck equation (70) takes the form

$$
\frac{\partial P}{\partial t}=\frac{\partial}{\partial J_{i}}\left[D_{i j}(\mathbf{J})\left(\frac{\partial P}{\partial J_{j}}+\beta m P \Omega_{j}(\mathbf{J})\right)\right],
$$

where $D_{i j}(\mathbf{J})$ is given by Eq. (111) with Eq. (116). Recalling that $\boldsymbol{\Omega}(\mathbf{J})=\partial H / \partial \mathbf{J}$, this equation is similar to the Kramers equation in Brownian theory [19]. This is a drift-diffusion equation describing the evolution of the distribution $P(\mathbf{J}, t)$ of the test particle in an "effective potential" $U_{\text {eff }}(\mathbf{J})=H(\mathbf{J})$ produced by the field particles. For 
$t \rightarrow+\infty$, the distribution of the test particle relaxes towards the Boltzmann distribution $P_{e}(\mathbf{J})=(A / N m) e^{-\beta m H(\mathbf{J})}$. Since the Fokker-Planck is valid at the order $1 / N$, the relaxation time scales like

$$
t_{R}^{b a t h} \sim N t_{D}
$$

If the field particles are in a stable steady state of the Vlasov equation that forbids any resonance (see Sect. IID), the diffusion tensor and the friction force can be simplified in a manner similar to that described in [67, 68]. In that case, the test particle still relaxes towards the distribution $f(\mathbf{J})$ of the bath on a timescale $t_{R}^{b a t h} \sim N t_{D}$. This timescale is shorter than the relaxation time of the system as a whole $t_{R}>N t_{D}$ so that the bath approximation, which assumes that the distribution of the field particles is "frozen", is justified.

\section{CONCLUSION}

In this paper, we have developed a kinetic theory of systems with long-range interactions which takes collective effects and spatial inhomogeneity into account. This improves our previous works [67, 68] where collective effects were neglected (leading to the Landau-type kinetic equation (59)) or taken into account in an unsatisfactory manner (making an ad hoc factorization assumption). The use of a biorthogonal basis of pairs of density-potential functions solves the shortcomings of our previous derivation. We recovered and confirmed the Lenard-Balescu-type kinetic equation (56) derived by Heyvaerts [69] from the Liouville equation, using the BBGKY hierarchy truncated at the level of the two-body distribution function (i.e. neglecting three-body correlations). Our derivation, starting from the Klimontovich equation and using a quasilinear approximation, may be technically simpler 107. Furthermore, it is completely parallel to the one exposed in [2, 72, 73] to derive the ordinary Lenard-Balescu equation for spatially homogeneous systems and in [51, 53] to derive a Lenard-Balescu-type equation appropriate to two-dimensional point vortices with axisymmetric distribution. Our approach therefore offers a unified description of kinetic theories for systems with long-range interactions. Interestingly, the same formalism can be used to describe the relaxation of a test particle in a bath of field particles through a Fokker-Planck equation. This is another advantage of our approach. We have obtained the expressions of the diffusion tensor and friction force directly from the equations of motion with angle-action variables, taking collective effects into account. We have shown that they can also be obtained from the Lenard-Balescu-type kinetic equation (56) by making a bath approximation leading to the Fokker-Planck equation (115). For simplicity of notation, we have considered a single species system, but the general case of a multi-species system can be treated easily and leads to the same results as those obtained by Heyvaerts [69].

The kinetic theory developed in this paper is valid at the order $1 / N$ so it describes the evolution of the system on a timescale $N t_{D}$. The dynamical evolution of the system is due to a condition of resonance between distant orbits. For spatially homogeneous one dimensional systems, there is no resonance so the Lenard-Balescu collision term vanishes (this result is well-known in plasma physics [49, 50] and it has been rediscovered recently for the HMF model [47, 48]). This implies that the relaxation time is larger than $N t_{D}$. In that case, we need to develop the kinetic theory at higher orders (taking three-body, four-body,... correlations into account). At present, no such theory exists and the scaling of the relaxation time with $N$ remains an open problem. We can try to make speculations based on available results of numerical simulations. The most natural scaling would be $N^{2} t_{D}$ which corresponds to the next order term in the expansion of the basic equations of the kinetic theory in powers of $1 / N$ [68]. An $N^{2}$ scaling is indeed observed numerically [84, 85] for spatially homogeneous one-dimensional plasmas (in that context, $N$ represents the number of charges in the Debye sphere). However, the scaling of the relaxation time may be more complex. For example, for the permanently spatially homogeneous HMF model, Campa et al. [86] report a relaxation time scaling like $e^{N} t_{D}$. This timescale is, however, questioned in recent works [87] who find a $N^{2} t_{D}$ scaling. This would be more natural on a theoretical point of view. It could also happen that the system never reaches statistical equilibrium, i.e. the evolution may be non-ergodic and the mixing by "collisions" inefficient. This may be the case for the two-dimensional point vortex gas when the profile of angular velocity is monotonic [51 53]. In that case, there is no resonance and the relaxation time is larger than $N t_{D}$. However, the precise scaling with $N$ is still an open problem, and even the relaxation towards the Boltzmann distribution is uncertain. The situation is different for spatially homogeneous systems in $d>1$ dimensions and for spatially inhomogeneous systems in any dimension (and for non-axisymmetric configurations of the point vortex gas). In these cases, there are potentially more resonances so the relaxation time can be reduced and achieve the natural scaling $N t_{D}$ corresponding to the first order of the kinetic theory 68]. An $N t_{D}$ scaling is indeed observed numerically for spatially inhomogeneous one dimensional stellar systems [66, 88 90] and for the spatially inhomogeneous HMF model [91]. On the other hand, for the HMF model, if an initially spatially homogeneous distribution function becomes Vlasov unstable during the collisional evolution, a dynamical phase transition from a non-magnetized to a magnetized state takes place (as theoretically studied in [92]) and the relaxation time could be intermediate between $N^{2} t_{D}$ (permanently homogeneous) and $N t_{D}$ (permanently inhomogeneous). In that situation, Yamaguchi et al. 93] find a relaxation time scaling like $N^{\delta} t_{D}$ with $\delta=1.7$. 
The previous argument (suggesting $1<\delta<2$ ) may provide a first step towards the explanation of this anomalous exponent.

The results of this paper also apply to self-gravitating systems that are, by essence, spatially inhomogeneous and limited in extension. The consideration of spatial inhomogeneity avoids the logarithmic divergence occurring at large scales when one makes the local approximation [70]. There remains, however, a logarithmic divergence at small scales due to the neglect of strong collisions in the kinetic theory. On the other hand, self-gravitating systems are very particular since no statistical equilibrium state exists in a strict sense (the entropy is not bounded from above) [3 [5]. Therefore, the Lenard-Balescu-type kinetic equation (56) does not relax towards a steady state. On the contrary, the system takes a "core-halo" structure and keeps evolving. The halo expands due to the evaporation of high energy stars [30] and, when the system becomes sufficiently centrally condensed, the core collapses as a result of the gravothermal catastrophe [31, 32] caused by the negative specific heat of the central region. This evolution is clearly illustrated in the numerical simulation of Cohn 28 based on the orbit-averaged Fokker-Planck equation. Core collapse ultimately leads to the formation of binary stars [27], followed by a post-collapse evolution [94], and gravothermal oscillations [95]. The formation of binary stars is not taken into account in the kinetic theory because it results from triple collisions (three-body correlations) and is of the strong interaction type. We also note that, since the Boltzmann distribution is not normalizable for self-gravitating systems (infinite mass problem), the thermal bath approximation that we have developed in Sec. IIIF is not justified. However, it could be easily generalized by replacing the Boltzmann distribution by the King model which takes evaporation into account and leads to a truncated Boltzmann distribution with a finite mass viewed as a metastable equilibrium state [5].

At the physical level, one interest of the kinetic theory is to show that the "collisional" evolution of the system is controlled by resonances encapsulated in a $\delta$-function. This explains the scaling of the relaxation time with $N$. For example, in the case of one dimensional potentials, the relaxation time of spatially homogeneous systems is larger than $N t_{D}$ because there is no resonance, while it is of order $N t_{D}$ for spatially inhomogeneous systems due to the arising of resonances. Obtaining this result was our original motivation to develop a kinetic theory for spatially inhomogeneous systems in [67, 68]. At a practical level, we believe that the Lenard-Balescu equation (566) with angle-action variables is very complicated to solve, so its practical interest is limited. With present-day computers, it is much easier to solve the $N$-body problem directly. Although the description of the motion becomes simple in angle-action variables, all the complexity is now embodied in the relation between position-momentum variables and angle-action variables. The usefulness of equation (56) is therefore limited to systems for which this relation can be established, either analytically or numerically. Some simplification can be gained if we restrict ourselves to spherically symmetric stellar systems as done by Heyvaerts [69] for stellar systems. But, even in that case the final kinetic equations remain complicated and approximations may be welcome to simplify the problem and lead to more tractable equations. The Fokker-Planck treatment that we have given here is one possible approximation. The restriction to one dimensional systems with simple potentials (e.g. the HMF model) is another one.

\section{Appendix A: Bare and dressed potentials of interaction}

We assume that the particles interact via a long-range binary potential $u\left(\left|\mathbf{r}-\mathbf{r}^{\prime}\right|\right)$ so that

$$
\Phi(\mathbf{r}, t)=\int u\left(\left|\mathbf{r}-\mathbf{r}^{\prime}\right|\right) f\left(\mathbf{r}^{\prime}, \mathbf{v}^{\prime}, t\right) d \mathbf{r}^{\prime} d \mathbf{v}^{\prime} .
$$

We introduce angle-action variables and decompose the potential of interaction in Fourier modes

$$
u\left(\left|\mathbf{r}(\mathbf{w}, \mathbf{J})-\mathbf{r}^{\prime}\left(\mathbf{w}^{\prime}, \mathbf{J}^{\prime}\right)\right|\right)=\sum_{\mathbf{k}, \mathbf{k}^{\prime}} e^{i\left(\mathbf{k} \cdot \mathbf{w}-\mathbf{k}^{\prime} \cdot \mathbf{w}^{\prime}\right)} A_{\mathbf{k}, \mathbf{k}^{\prime}}\left(\mathbf{J}, \mathbf{J}^{\prime}\right),
$$

where the Fourier coefficients are given by

$$
A_{\mathbf{k}, \mathbf{k}^{\prime}}\left(\mathbf{J}, \mathbf{J}^{\prime}\right)=\frac{1}{(2 \pi)^{2 d}} \int u\left(\left|\mathbf{r}(\mathbf{w}, \mathbf{J})-\mathbf{r}^{\prime}\left(\mathbf{w}^{\prime}, \mathbf{J}^{\prime}\right)\right|\right) e^{-i\left(\mathbf{k} \cdot \mathbf{w}-\mathbf{k}^{\prime} \cdot \mathbf{w}^{\prime}\right)} d \mathbf{w} d \mathbf{w}^{\prime} .
$$

The fluctuations of the potential are related to the fluctuations of the distribution function by

$$
\delta \Phi(\mathbf{w}, \mathbf{J}, t)=\int u\left(\left|\mathbf{r}-\mathbf{r}^{\prime}\right|\right) \delta f\left(\mathbf{w}^{\prime}, \mathbf{J}^{\prime}, t\right) d \mathbf{w}^{\prime} d \mathbf{J}^{\prime},
$$

where we have used the identity $d \mathbf{r}^{\prime} d \mathbf{v}^{\prime}=d \mathbf{w}^{\prime} d \mathbf{J}^{\prime}$ valid for a canonical change of variables. Taking the Fourier-Laplace transform of this expression, and using Eqs. (18) and (A3), we get

$$
\delta \tilde{\Phi}(\mathbf{k}, \mathbf{J}, \omega)=(2 \pi)^{d} \sum_{\mathbf{k}^{\prime}} \int d \mathbf{J}^{\prime} A_{\mathbf{k}, \mathbf{k}^{\prime}}\left(\mathbf{J}, \mathbf{J}^{\prime}\right) \delta \tilde{f}\left(\mathbf{k}^{\prime}, \mathbf{J}^{\prime}, \omega\right) .
$$


Substituting Eq. (21) in Eq. (A5), we obtain the Fredholm integral equation

$$
\delta \tilde{\Phi}(\mathbf{k}, \mathbf{J}, \omega)-(2 \pi)^{d} \sum_{\mathbf{k}^{\prime}} \int d \mathbf{J}^{\prime} A_{\mathbf{k}, \mathbf{k}^{\prime}}\left(\mathbf{J}, \mathbf{J}^{\prime}\right) \frac{\mathbf{k}^{\prime} \cdot \frac{\partial f^{\prime}}{\partial \mathbf{J}^{\prime}}}{\mathbf{k}^{\prime} \cdot \boldsymbol{\Omega}^{\prime}-\omega} \delta \tilde{\Phi}\left(\mathbf{k}^{\prime}, \mathbf{J}^{\prime}, \omega\right)=(2 \pi)^{d} \sum_{\mathbf{k}^{\prime}} \int d \mathbf{J}^{\prime} A_{\mathbf{k}, \mathbf{k}^{\prime}}\left(\mathbf{J}, \mathbf{J}^{\prime}\right) \frac{\delta \hat{f}\left(\mathbf{k}^{\prime}, \mathbf{J}^{\prime}, 0\right)}{i\left(\mathbf{k}^{\prime} \cdot \mathbf{\Omega}^{\prime}-\omega\right)} .
$$

This equation relates the Fourier-Laplace transform of the fluctuations of the potential to the Fourier transform of the initial fluctuations of the distribution function. It is therefore equivalent to Eq. (37). When collective effects are neglected, the foregoing expression reduces to

$$
\delta \tilde{\Phi}(\mathbf{k}, \mathbf{J}, \omega)=(2 \pi)^{d} \sum_{\mathbf{k}^{\prime}} \int d \mathbf{J}^{\prime} A_{\mathbf{k}, \mathbf{k}^{\prime}}\left(\mathbf{J}, \mathbf{J}^{\prime}\right) \frac{\delta \hat{f}\left(\mathbf{k}^{\prime}, \mathbf{J}^{\prime}, 0\right)}{i\left(\mathbf{k}^{\prime} \cdot \mathbf{\Omega}^{\prime}-\omega\right)} .
$$

Comparing this expression with Eq. (37), we see that $-1 / D_{\mathbf{k}, \mathbf{k}^{\prime}}\left(\mathbf{J}, \mathbf{J}^{\prime}, \omega\right)_{\text {bare }}$ is just the Fourier transform $A_{\mathbf{k}, \mathbf{k}^{\prime}}\left(\mathbf{J}, \mathbf{J}^{\prime}\right)$ of the "bare" potential of interaction $u$ with angle-action variables. When collective effects are taken into account, Eq. (A7) is replaced by Eq. (37) where according to (A6) the "dressed" potential of interaction satisfies

$$
\frac{1}{D_{\mathbf{k}, \mathbf{k}^{\prime}}\left(\mathbf{J}, \mathbf{J}^{\prime}, \omega\right)}-(2 \pi)^{d} \sum_{\mathbf{k}^{\prime \prime}} \int d \mathbf{J}^{\prime \prime} A_{\mathbf{k}, \mathbf{k}^{\prime \prime}}\left(\mathbf{J}, \mathbf{J}^{\prime \prime}\right) \frac{\mathbf{k}^{\prime \prime} \cdot \frac{\partial f^{\prime \prime}}{\partial \mathbf{J}^{\prime \prime}}}{\mathbf{k}^{\prime \prime} \cdot \boldsymbol{\Omega}^{\prime \prime}-\omega} \frac{1}{D_{\mathbf{k}^{\prime \prime}, \mathbf{k}^{\prime}}\left(\mathbf{J}^{\prime \prime}, \mathbf{J}^{\prime}, \omega\right)}=-A_{\mathbf{k}, \mathbf{k}^{\prime}}\left(\mathbf{J}, \mathbf{J}^{\prime}\right) .
$$

\section{Appendix B: The resolvant}

Substituting Eq. (37) in Eq. (21), we obtain

$$
\delta \tilde{f}(\mathbf{k}, \mathbf{J}, \omega)=-\frac{\mathbf{k} \cdot \frac{\partial f}{\partial \mathbf{J}}}{\mathbf{k} \cdot \boldsymbol{\Omega}-\omega}(2 \pi)^{d} \sum_{\mathbf{k}^{\prime \prime}} \int d \mathbf{J}^{\prime \prime} \frac{1}{D_{\mathbf{k}, \mathbf{k}^{\prime \prime}}\left(\mathbf{J}, \mathbf{J}^{\prime \prime}, \omega\right)} \frac{\delta \hat{f}\left(\mathbf{k}^{\prime \prime}, \mathbf{J}^{\prime \prime}, 0\right)}{i\left(\mathbf{k}^{\prime \prime} \cdot \mathbf{\Omega}^{\prime \prime}-\omega\right)}+\frac{\delta \hat{f}(\mathbf{k}, \mathbf{J}, 0)}{i(\mathbf{k} \cdot \boldsymbol{\Omega}-\omega)} .
$$

This equation relates the Fourier-Laplace transform of the fluctuations of the distribution function to the Fourier transform of the initial fluctuations of the distribution function. It can be written

$$
\delta \tilde{f}(\mathbf{k}, \mathbf{J}, \omega)=\sum_{\mathbf{k}^{\prime \prime}} \int d \mathbf{J}^{\prime \prime} R_{\mathbf{k}, \mathbf{k}^{\prime \prime}}\left(\mathbf{J}, \mathbf{J}^{\prime \prime}, \omega\right) \delta \hat{f}\left(\mathbf{k}^{\prime \prime}, \mathbf{J}^{\prime \prime}, 0\right),
$$

where

$$
R_{\mathbf{k}, \mathbf{k}^{\prime \prime}}\left(\mathbf{J}, \mathbf{J}^{\prime \prime}, \omega\right)=-\frac{\mathbf{k} \cdot \frac{\partial f}{\partial \mathbf{J}}}{\mathbf{k} \cdot \boldsymbol{\Omega}-\omega}(2 \pi)^{d} \frac{1}{D_{\mathbf{k}, \mathbf{k}^{\prime \prime}}\left(\mathbf{J}, \mathbf{J}^{\prime \prime}, \omega\right)} \frac{1}{i\left(\mathbf{k}^{\prime \prime} \cdot \mathbf{\Omega}^{\prime \prime}-\omega\right)}+\frac{\delta\left(\mathbf{J}-\mathbf{J}^{\prime \prime}\right)}{i(\mathbf{k} \cdot \boldsymbol{\Omega}-\omega)} \delta_{\mathbf{k}, \mathbf{k}^{\prime \prime}}
$$

is called the resolvant. If we consider an initial condition of the form $\delta f(\mathbf{w}, \mathbf{J}, 0)=m \delta\left(\mathbf{w}-\mathbf{w}^{\prime}\right) \delta\left(\mathbf{J}-\mathbf{J}^{\prime}\right)$ implying

$$
\delta \hat{f}(\mathbf{k}, \mathbf{J}, 0)=\frac{m}{(2 \pi)^{d}} e^{-i \mathbf{k} \cdot \mathbf{w}^{\prime}} \delta\left(\mathbf{J}-\mathbf{J}^{\prime}\right)
$$

we find that

$$
\delta \tilde{f}(\mathbf{k}, \mathbf{J}, \omega)=-\frac{\mathbf{k} \cdot \frac{\partial f}{\partial \mathbf{J}}}{\mathbf{k} \cdot \boldsymbol{\Omega}-\omega} \sum_{\mathbf{k}^{\prime \prime}} m e^{-i \mathbf{k}^{\prime \prime} \cdot \mathbf{w}^{\prime}} \frac{1}{D_{\mathbf{k}, \mathbf{k}^{\prime \prime}}\left(\mathbf{J}, \mathbf{J}^{\prime}, \omega\right)} \frac{1}{i\left(\mathbf{k}^{\prime \prime} \cdot \boldsymbol{\Omega}^{\prime}-\omega\right)}+\frac{m}{(2 \pi)^{d}} e^{-i \mathbf{k} \cdot \mathbf{w}^{\prime}} \delta\left(\mathbf{J}-\mathbf{J}^{\prime}\right) \frac{1}{i(\mathbf{k} \cdot \boldsymbol{\Omega}-\omega)} .
$$

This quantity is called the propagator of the linearized Vlasov equation. If we substitute Eq. (A5) in Eq. (21), we obtain the integral equation

$$
\delta \tilde{f}(\mathbf{k}, \mathbf{J}, \omega)=\frac{\mathbf{k} \cdot \frac{\partial f}{\partial \mathbf{J}}}{\mathbf{k} \cdot \boldsymbol{\Omega}-\omega}(2 \pi)^{d} \sum_{\mathbf{k}^{\prime}} \int d \mathbf{J}^{\prime} A_{\mathbf{k}, \mathbf{k}^{\prime}}\left(\mathbf{J}, \mathbf{J}^{\prime}\right) \delta \tilde{f}\left(\mathbf{k}^{\prime}, \mathbf{J}^{\prime}, \omega\right)+\frac{\delta \hat{f}(\mathbf{k}, \mathbf{J}, 0)}{i(\mathbf{k} \cdot \boldsymbol{\Omega}-\omega)} .
$$

This equation relates the Fourier-Laplace transform of the fluctuations of the distribution function to the Fourier transform of the initial fluctuations of the distribution function, so it is equivalent to Eq. (B1). 


\section{Appendix C: Auto-correlation of the fluctuations of the one-particle distribution}

According to Eq. (20), we have

$$
\begin{aligned}
\left\langle\delta \hat{f}(\mathbf{k}, \mathbf{J}, 0) \delta \hat{f}\left(\mathbf{k}^{\prime}, \mathbf{J}^{\prime}, 0\right)\right\rangle & =\int \frac{d \mathbf{w}}{(2 \pi)^{d}} \frac{d \mathbf{w}^{\prime}}{(2 \pi)^{d}} e^{-i\left(\mathbf{k} \cdot \mathbf{w}+\mathbf{k}^{\prime} \cdot \mathbf{w}^{\prime}\right)}\left\langle\delta f(\mathbf{w}, \mathbf{J}, 0) \delta f\left(\mathbf{w}^{\prime}, \mathbf{J}^{\prime}, 0\right)\right\rangle \\
& =\int \frac{d \mathbf{w}}{(2 \pi)^{d}} \frac{d \mathbf{w}^{\prime}}{(2 \pi)^{d}} e^{-i\left(\mathbf{k} \cdot \mathbf{w}+\mathbf{k}^{\prime} \cdot \mathbf{w}^{\prime}\right)}\left[\left\langle f_{d}(\mathbf{w}, \mathbf{J}, 0) f_{d}\left(\mathbf{w}^{\prime}, \mathbf{J}, 0\right)\right\rangle-f(\mathbf{J}) f\left(\mathbf{J}^{\prime}\right)\right] .
\end{aligned}
$$

The expression of the discrete distribution function recalled in the first paragraph of Sec. [A leads to

$$
\begin{aligned}
\left\langle f_{d}(\mathbf{w}, \mathbf{J}, 0) f_{d}\left(\mathbf{w}^{\prime}, \mathbf{J}^{\prime}, 0\right)\right\rangle & =m^{2} \sum_{i, j}\left\langle\delta\left(\mathbf{w}-\mathbf{w}_{i}\right) \delta\left(\mathbf{J}-\mathbf{J}_{i}\right) \delta\left(\mathbf{w}^{\prime}-\mathbf{w}_{j}\right) \delta\left(\mathbf{J}^{\prime}-\mathbf{J}_{j}\right)\right\rangle \\
& =m^{2} \sum_{i}\left\langle\delta\left(\mathbf{w}-\mathbf{w}_{i}\right) \delta\left(\mathbf{J}-\mathbf{J}_{i}\right) \delta\left(\mathbf{w}-\mathbf{w}^{\prime}\right) \delta\left(\mathbf{J}-\mathbf{J}^{\prime}\right)\right\rangle \\
& +m^{2} \sum_{i \neq j}\left\langle\delta\left(\mathbf{w}-\mathbf{w}_{i}\right) \delta\left(\mathbf{J}-\mathbf{J}_{i}\right) \delta\left(\mathbf{w}^{\prime}-\mathbf{w}_{j}\right) \delta\left(\mathbf{J}^{\prime}-\mathbf{J}_{j}\right)\right\rangle \\
& =m f(\mathbf{J}) \delta\left(\mathbf{w}-\mathbf{w}^{\prime}\right) \delta\left(\mathbf{J}-\mathbf{J}^{\prime}\right)+f(\mathbf{J}) f\left(\mathbf{J}^{\prime}\right),
\end{aligned}
$$

where we have assumed that there is no correlation initially (if there are initial correlations, it can be shown that they are washed out rapidly [72] so they have no effect on the final form of the collision term). Combining Eqs. (C1) and (C2), we obtain

$$
\left\langle\delta \hat{f}(\mathbf{k}, \mathbf{J}, 0) \delta \hat{f}\left(\mathbf{k}^{\prime}, \mathbf{J}^{\prime}, 0\right)\right\rangle=\frac{1}{(2 \pi)^{d}} \delta_{\mathbf{k},-\mathbf{k}^{\prime}} \delta\left(\mathbf{J}-\mathbf{J}^{\prime}\right) m f(\mathbf{J})
$$

[1] Dynamics and Thermodynamics of Systems with Long-Range Interactions, edited by T. Dauxois, S. Ruffo, E. Arimondo and M. Wilkens, Lectures Notes in Physics 602 (Berlin: Springer, 2002)

[2] A. Campa, T. Dauxois, S. Ruffo, Physics Reports 480, 57 (2009)

[3] T. Padmanabhan, Phys. Rep. 188, 285 (1990)

[4] J. Katz, Found. Phys. 33, 223 (2003)

[5] P.H. Chavanis, Int. J. Mod. Phys. B 20, 3113 (2006)

[6] L. Boltzmann, Wien, Ber. 66, 275 (1872)

[7] L.D. Landau, Phys. Z. Sowj. Union 10, 154 (1936)

[8] P. Debye, E. Hückel, Phys. Z. 24, 305 (1923)

[9] A. Lenard, Ann. Phys. (N.Y.) 10, 390 (1960)

[10] R. Balescu, Phys. Fluids 3, 52 (1960)

[11] J. Hubbard, Proc. R. Soc. Lond. 260, 114 (1961)

[12] S. Chandrasekhar, Principles of Stellar Dynamics (University of Chicago press, 1942)

[13] S. Chandrasekhar, Astrophys. J. 97, 255 (1943)

[14] S. Chandrasekhar, Astrophys. J. 97, 263 (1943)

[15] S. Chandrasekhar, J. von Neumann, Astrophys. J. 95, 489 (1942)

[16] R.S. Cohen, L. Spitzer, P.M. Routly, Phys. Rev. 80, 230 (1950)

[17] J.H. Jeans, Astronomy and Cosmogony (Cambridge Univ. Press, 1929)

[18] S. Chandrasekhar, Rev. Mod. Phys. 21, 383 (1949)

[19] H.A. Kramers, Physica 7, 284 (1940)

[20] L. Spitzer, R. Härm, Astrophys. J. 127, 544 (1958)

[21] R. Michie, Mon. not. R. astron. Soc. 125, 127 (1963)

[22] I.R. King, Astron. J. 70, 376 (1965)

[23] M. Lemou, P.H. Chavanis, Physica A 389, 1021 (2010)

[24] I. King, Astron. J. 65, 122 (1960)

[25] J.H. Jeans, Mon. not. R. astron. Soc. 76, 71 (1915)

[26] A.A. Vlasov, Zh. Eksp. i Teor. Fiz. 8, 291 (1938)

[27] M. Hénon, Ann. Astrophys. 24, 369 (1961)

[28] H. Cohn, Astrophys. J. 242, 765 (1980)

[29] C. Lancellotti, M. Kiessling, Astrophys. J. 549, L93 (2001)

[30] L. Spitzer, Mon. not. R. astron. Soc. 100, 396 (1940) 
[31] V.A. Antonov, Vest. Leningr. Gos. Univ. 7, 135 (1962).

[32] D. Lynden-Bell, R. Wood, Mon. not. R. astron. Soc. 138, 495 (1968)

[33] G. Severne, M.J. Haggerty, Astro. Space Sci. 45, 287 (1976)

[34] J.P. Parisot, G. Severne, Astro. Space Sci. 61, 121 (1979)

[35] H. Kandrup, Astrophys. J. 244, 316 (1981)

[36] P.H. Chavanis, Physica A 387, 787 (2008)

[37] P.H. Chavanis, Physica A 387, 1504 (2008)

[38] P.H. Chavanis, Physica A 361, 81 (2006)

[39] R.M. Thorne, Astrophys. J. 151, 671 (1968)

[40] R.H. Miller, Astrophys. J. 151, 679 (1968)

[41] I.H. Gilbert, Astrophys. J. 152, 1043 (1968)

[42] I.H. Gilbert, Astrophys. J. 159, 239 (1970)

[43] I. Lerche, Astrophys. J. 166, 207 (1971)

[44] M.D. Weinberg, Astrophys. J. 410, 543 (1993)

[45] M. Antoni, S. Ruffo, Phys. Rev. E 52, 2361 (1995)

[46] C. Pichon, Ph.D. thesis, Cambridge (1994)

[47] P.H. Chavanis, J. Vatteville, F. Bouchet, Eur. Phys. J. B 46, 61 (2005)

[48] F. Bouchet, T. Dauxois, Phys. Rev. E 72, 045103 (2005)

[49] O.C. Eldridge, M. Feix, Phys. Fluids. 6, 398 (1962)

[50] B.B. Kadomtsev, O.P. Pogutse, Phys. Rev. Lett. 25, 1155 (1970)

[51] D. Dubin, T.M. O'Neil, Phys. Rev. Lett. 60, 1286 (1988)

[52] P.H. Chavanis, Phys. Rev. E 64, 026309 (2001)

[53] P.H. Chavanis, arXiv:1107.1447

[54] A. Kalnajs, Astrophys. J. 212, 637 (1977)

[55] J. Goodman, Astrophys. J. 329, 612 (1988)

[56] M.D. Weinberg, Astrophys. J. 368, 66 (1991)

[57] C. Pichon, R.C. Cannon, Mon. Not. R. Astron. Soc. 291, 616 (1997)

[58] P. Valageas, Astron. Astrophys. 450, 445 (2006)

[59] D. Lynden-Bell, A.J. Kalnajs, Mon. Not. R. Astron. Soc. 157, 1 (1972)

[60] S. Tremaine, M. Weinberg, Mon. not. R. astron. Soc. 209, 729 (1984)

[61] J. Binney, C. Lacey, Mon. not. R. astron. Soc. 230, 597 (1988)

[62] M.D. Weinberg, Mon. Not. R. Astron. Soc. 297, 101 (1998)

[63] R.W. Nelson, S. Tremaine, Mon. not. R. astron. Soc. 306, 1 (1999)

[64] M.D. Weinberg, Mon. Not. R. Astron. Soc. 328, 311 (2001)

[65] C. Pichon, D. Aubert, Mon. Not. R. Astron. Soc. 368, 1657 (2006)

[66] P. Valageas, Phys. Rev. E 74, 016606 (2006)

[67] P.H. Chavanis, Physica A 377, 469 (2007)

[68] P.H. Chavanis, J. Stat. Mech (2010) P05019

[69] J. Heyvaerts, Mon. Not. R. Astron. Soc. 407, 355 (2010)

[70] J. Binney, S. Tremaine, Galactic Dynamics (Princeton Series in Astrophysics, 2008)

[71] A. Kalnajs, Astrophys. J. 166, 275 (1971)

[72] E.M. Lifshitz, L.P. Pitaevskii, Physical Kinetics (Pergamon Press, Oxford, 1981)

[73] P.H. Chavanis, arXiv:1112.0772

[74] C.P Ma, E. Bertschinger, Astrophys. J. 612, 28 (2004)

[75] Yu. L. Klimontovich, The Statistical Theory of Non-Equilibrium Processes in a Plasma (MIT press, Cambridge Massachusetts, 1967)

[76] H. Goldstein, Classical Mechanics (Addison-Wesley, Cambridge MA, 1956)

[77] D. Lynden-Bell, Mon. Not. R. Astron. Soc. 136, 101 (1967)

[78] J. Barré, A. Olivetti, Y.Y. Yamaguchi, J. Stat. Mech. (2010) P08002

[79] J. Barré, A. Olivetti, Y.Y. Yamaguchi, arXiv:1104.1890.

[80] F. Bouchet, S. Gupta, D. Mukamel, Physica A 389, 4389 (2010)

[81] H. Risken, The Fokker-Planck equation (Springer, 1989)

[82] B.D. Fried, in Plasma Physics in Theory and Application, edited by W.B. Kunkel (Mc Graw-Hill, New York, 1966)

[83] S. Ichimaru, Basic Principles of Plasma Physics (Westview, 1973)

[84] J. Dawson, Phys. Fluids 7, 419 (1964)

[85] J.L. Rouet, M. Feix, Phys. Fluids B 3, 1830 (1991)

[86] A. Campa, A. Giansanti, G. Morelli, Phys. Rev. E 76, 041117 (2007)

[87] S. Gupta, S. Ruffo, private communication

[88] B.N. Miller, Phys. Rev. E 53, R4279 (1996)

[89] T. Tsuchiya, N. Gouda, T. Konishi, Phys. Rev. E 53, 2210 (1996)

[90] M. Joyce, T. Worrakitpoonpon, J. Stat. Mech. 10, 12 (2010)

[91] P. de Buyl, D. Mukamel, S. Ruffo, arXiv:1012.2594]

[92] A. Campa, P.H. Chavanis, A. Giansanti, G. Morelli, Phys. Rev. E 78, 040102 (2008)

[93] Y. Yamaguchi, J. Barré, F. Bouchet, T. Dauxois, S. Ruffo, Physica A 337, 36 (2004) 
[94] S. Inagaki, D. Lynden-Bell, Mon. Not. R. Astron. Soc. 205, 913 (1983)

[95] D. Sugimoto, E. Bettwieser, Mon. Not. R. Astron. Soc. 204, 19 (1983)

[96] Indeed, Chandrasekhar [13, 14] models the evolution of globular clusters by the Kramers [19] equation which has a fixed temperature (canonical description) while a more relevant kinetic equation would be the Landau [7] equation which conserves energy (microcanonical description).

[97] The orbit-averaged approximation has been recently criticized by Lancellotti and Kiessling [29].

[98] There remains, however, a logarithmic divergence at small scales due to the neglect of strong collisions.

[99] In a plasma, since the Coulomb force between electrons is repulsive, each particle of the plasma tends to attract to it particles of opposite charge and to repel particles of like charge, thereby creating a kind of "cloud" of opposite charge which screens the interaction at the scale of the Debye length. In the gravitational case, since the Newton force is attractive, the situation is considerably different. The test star draws neighboring stars into its vicinity and these add their gravitational force to that of the test star itself. The "bare" gravitatonal force of the test star is thus augmented rather than shielded. The polarization acts to increase the effecive gravitational mass of a test star.

[100] This model [45] is directly inspired by astrophysics. It was proposed by Pichon [46] as a simplified model to describe the formation of bars in disk galaxies (for an historic of the HMF model, see [4]]).

[101] We considered 1D systems to simplify the notations but the formal generalization in $d$ dimensions is straightforward.

[102] The conventional Laplace transform uses $-s$ in place of $-i \omega$ in the integrand. However, we find it more convenient to use this latter notation in order to make the link with the case where the perturbations are decomposed in plane waves with pulsation $\omega$ and wavenumber $\mathbf{k}$ (e.g. when studying the Vlasov stability problem and obtaining the dispersion relation). The notation $-i \omega$ for the Laplace transform is relatively standard in kinetic theory.

[103] The dispersion relation associated with the linearized Vlasov equation corresponds to det $(\epsilon(\omega))=0$. The Vlasov dynamical stability of self-gravitating systems has been studied in [54 58]. For the HMF model, $\epsilon(\omega)$ is a $2 \times 2$ matrix. In that case, the evolution of perturbations governed by the linearized Vlasov equation has been studied by [78, 79] who evidenced an interesting situation of algebraic damping.

[104] This results from Eqs. (25), (35), and (A25) of Heyvaerts [69].

[105] The Markovian approximation may not be justified in every situation. For example, the (exponential) decay rate of the force auto-correlation function diverges close to a critical point [38] making this approximation invalid.

[106] For very particular situations such that $\mathbf{k}^{\prime} \cdot \boldsymbol{\Omega}\left(\mathbf{J}^{\prime}\right) \neq \mathbf{k} \cdot \boldsymbol{\Omega}(\mathbf{J})$ for any $\left(\mathbf{k}^{\prime}, \mathbf{J}^{\prime}\right) \neq(\mathbf{k}, \mathbf{J})$ (no resonance), the relaxation time is larger than $N t_{D}$.

[107] Of course, it is well-known that the approach based on the first two equations of the BBGKY hierarchy (neglecting three-body correlations) is equivalent to the approach starting from the Klimontovich equation and making a quasilinear approximation. More generally, the whole BBGKY hierarchy can be reconstructed from the Klimontovich equation 82, 83]. However, it has been acknowledged in plasma physics that the Klimontovich approach provides a technically simpler and more transparent derivation of the Lenard-Balescu equation than the one starting from the BBGKY hierarchy [75, 82]. 